\title{
WestVirginiaUniversity
}

THE RESEARCH REPOSITORY @ WVU

Graduate Theses, Dissertations, and Problem Reports

2006

\section{Religiousness, spirituality, and emotional salience in decision- making processes and outcomes}

\author{
Loriann Sonntag \\ West Virginia University
}

Follow this and additional works at: https://researchrepository.wvu.edu/etd

\section{Recommended Citation}

Sonntag, Loriann, "Religiousness, spirituality, and emotional salience in decision-making processes and outcomes" (2006). Graduate Theses, Dissertations, and Problem Reports. 2370.

https://researchrepository.wvu.edu/etd/2370

This Thesis is protected by copyright and/or related rights. It has been brought to you by the The Research Repository @ WVU with permission from the rights-holder(s). You are free to use this Thesis in any way that is permitted by the copyright and related rights legislation that applies to your use. For other uses you must obtain permission from the rights-holder(s) directly, unless additional rights are indicated by a Creative Commons license in the record and/ or on the work itself. This Thesis has been accepted for inclusion in WVU Graduate Theses, Dissertations, and Problem Reports collection by an authorized administrator of The Research Repository @ WVU. For more information, please contact researchrepository@mail.wvu.edu. 


\title{
Religiousness, Spirituality, and Emotional Salience in
} Decision-Making Processes and Outcomes

\author{
Loriann Sonntag
}

Thesis submitted to the Eberly College of Arts and Sciences in partial fulfillment of the requirements for the degree of

\author{
Master of Science \\ in \\ Psychology
}

\author{
Julie Hicks Patrick, Ph.D., Chair \\ Kevin T. Larkin, Ph.D. \\ JoNell Strough, Ph.D. \\ Department of Psychology \\ Morgantown, West Virginia \\ 2006
}

Keywords: Decision-Making, Problem-Solving, End-of-Life Decisions, Religiousness, Spirituality, Emotional Salience

Copyright 2006 Loriann Sonntag 


\section{ABSTRACT \\ Religiousness, Spirituality, and Emotional Salience in Decision-Making Processes and Outcomes \\ Loriann Sonntag}

The associations of religiousness and spirituality in decision-making processes and outcomes were investigated. One hundred younger adults $(M$ age $=19.9, S D=1.5)$ and seventy-eight older adults $(M$ age $=71.1, S D=7.9)$ completed self-administered surveys including vignettes varying in emotional salience. Responses were coded for problem-solving strategies and decisional outcomes. ANCOVA results indicate that the choice of strategies varies depending on the age and level of spirituality of the individual as well as the domain of the problem. Results from a multinomial logistic regression indicate that end-of-life decisions vary as a function of both age and religiousness $\left[X^{2}(4\right.$, $N=163)=9.34, p=.05$ and $\chi^{2}(4, N=163)=11.33, p<.05$ respectively]. However, the effects of religiousness and spirituality are not pervasive across the three decisional domains. Results are discussed in terms of the importance of linking problem-solving strategies and decision-making outcomes. 


\section{Dedication}

I would like to dedicate this thesis to my husband Scott, whose endless love and support offered me the encouragement I needed when I was ready to give up; to my three daughters, Crystal, Caitlyn, and Cirsten, who gave up Minnesota so Mom could follow her dreams; to my parents, the Pingels and the Sonntags, for their "long-distance" words of encouragement; and to my dear friend Joy who helped me to see my true potential. 


\section{Acknowledgements}

I would first like to thank Dr. Julie Patrick, my advisor and chair of my committee, for her guidance, support, and humor in helping me to finish my thesis. Her teaching efforts, and patience with my constant questions, have been an inspiration. I would also like to thank Dr. Stan Cohen, Dr. Kevin Larkin, and Dr. JoNell Strough for serving on my thesis committee. Their guidance and suggestions contributed greatly to this project. Lastly, I would like to thank Brian Ayotte for his support and encouragement as a student colleague and his patience with all of my statistics and SPSS questions. My appreciation and thanks goes out to each of these individuals for their guidance and support in my endeavors. 


\section{Table of Contents}

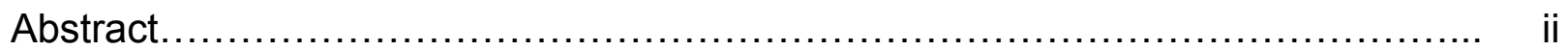

Chapter 1: Problem Statement................................................... 1

Chapter 2: Introduction.......................................................... 5

Problem-Solving Strategies.................................................. 5

Decision-Making Outcomes........................................... 8

Decision-Making Processes and Outcomes............................... 9

Emotional Salience ....................................................... 11

Religiousness and Spirituality............................................ 12

Hypothetical End-of-Life Decisions....................................... 15

Summary of Current Study ............................................... 17

Research Questions and Hypotheses................................... 19

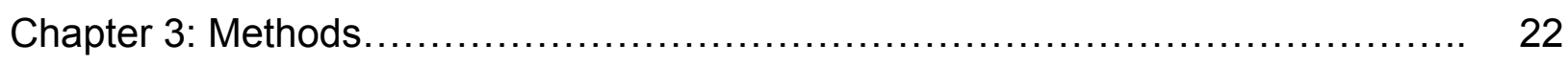

Participants.................................................................... 22

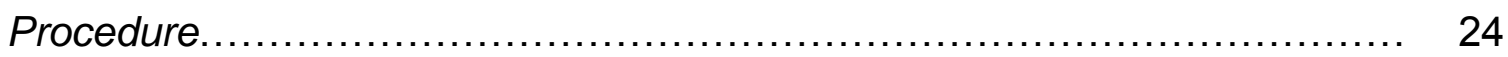

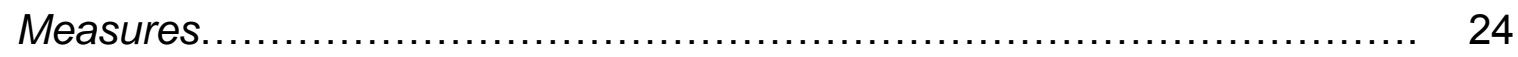

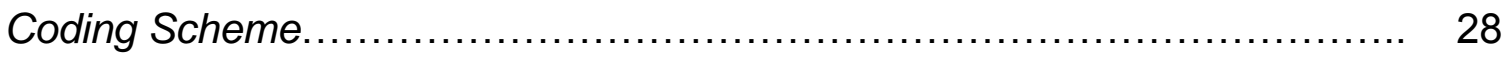

Problem-Solving Strategies....................................... 29

Decisional Outcomes........................................... 32

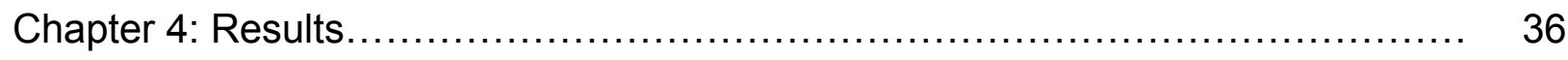

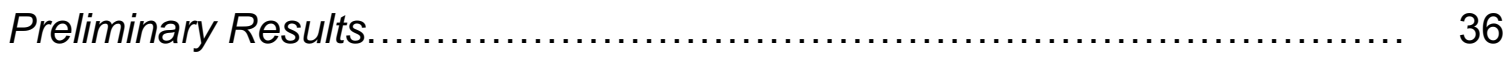

Descriptive Analyses................................................ 36

Correlational Analyses............................................. 37 
Hypothesis Testing .................................................... 38

End-of-Life Decisional Outcomes................................... 38

Religiousness, Spirituality, and Strategies.......................... 42

Exploratory Research Question........................................ 52

Chapter 5: Discussion......................................................... 55

Overview of the Study .................................................. 55

Specific Results and Discussion....................................... 56

Predictors of End-of-Life Decisions................................ 56

Problem-Solving Strategies and Emotional Salience................... 60

Decision-Making Processes and Outcomes.......................... 64

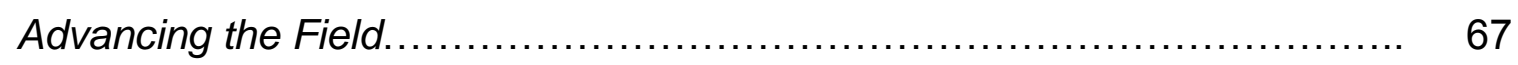

Religiousness and Spirituality .................................... 67

Everyday Problem Solving and End-of-Life Decisions.................. 69

Limitations....................................................................... 70

Conclusions and Future Directions....................................... $\quad 74$

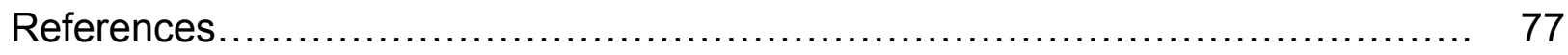

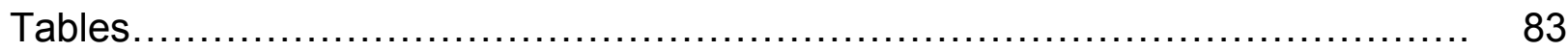

Table 1: Demographic Differences....................................... 83

Table 2: Group Differences on Study Variables.............................. 85

Table 3: Pearson Correlation Coefficients................................ 86

Table 4: Point Biserial and Phi Coefficients................................ 87

Table 5: End-of-Life Decisional Outcomes_Younger and Older Adults....... 88

Table 6: End-of-Life Decisional Outcomes_Within-Group Differences........ 89 
Table 7: Multinomial Logistic Regression................................... 90

Table $8: 2$ x 3 x 7 ANCOVA........................................... 92

Table 9: Domain x Strategy........................................... 94

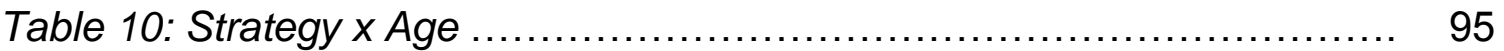

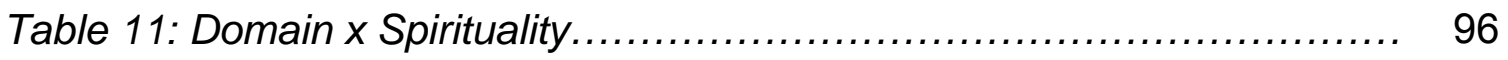

Table 12: Percentage of Strategies Within Each Domain..................... 98

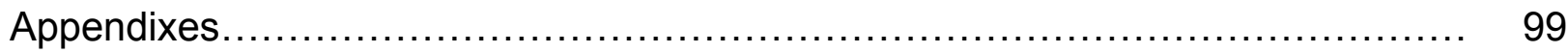

Appendix A: Informed Consent_Younger Adults........................... 99

Appendix B: Cover Letter ................................................ 101

Appendix C: Informed Consent_Older Adults.............................. 102

Appendix D: Decision Vignettes............................................ 104

Appendix E: Emotional Salience Pilot Study................................ 105

Appendix F: Demographics Questionnaire ................................ 109

Appendix G: Self-Assessed Health Scale................................. 111

Appendix H: Organizational Religiousness Scale........................... 112

Appendix I: STS_Universality Subscale ................................. 113

Appendix J: Coding Sheet................................................. 115

Appendix K: Strategies/Outcomes Coding Manual........................... 116 


\section{Chapter 1: Problem Statement}

To facilitate the understanding of the factors that influence an adult's decisionmaking process, researchers often present specific problem-solving situations using hypothetical vignettes (e.g., Blanchard-Fields, Jahnke, \& Camp, 1995; Cornelius \& Caspi, 1987; Denney \& Pearce, 1989; Patrick \& Strough, 2004). Participants are asked to respond to the vignettes by indicating what they would do, or what the target person should do, in each of the hypothetical situations. Problem-solving studies using vignettes generally focus on the strategies generated by the participants to solve the hypothetical problem. Examining problem-solving strategies is important because empirical research has shown consistent age differences in both the number and the type of strategies adults use to solve the problems presented (Berg, Meegan, \& Klaczynski, 1999; Blanchard-Fields et al., 1995; Cornelius \& Caspi, 1987; Denney \& Pearce, 1989; Watson \& Blanchard-Fields, 1998). In contrast, decision-making studies generally ignore the strategies and focus only on the nature of the decisional outcome. Research in this area suggests that age differences exist in the type of decisions adults of different ages make, with a strong influence of individual difference variables such as intelligence, personality (Willis, Dolan, \& Bertrand, 1999) and religiousness (Cicirelli, 1997a, 1998).

In addition to age, strategies and outcomes differ as a function of the nature of the problem domain. Blanchard-Fields et al. (1995) extended the use of hypothetical vignettes to examine whether the emotional salience of a problem influenced the types of strategies used in everyday problem-solving. The vignettes represented different problem domains and were rated as eliciting different levels of emotional salience-low, 
medium, or high. Results of this study demonstrated that the type of strategies an individual uses in response to a problem situation varies depending on the age of the individual, the problem domain, and the level of emotion elicited by the problem. Although emotional salience has been addressed in past studies, researchers examining the factors involved in decision-making processes and outcomes have virtually ignored the influence of religiousness and spirituality. When discussing the importance of religiousness and spirituality, existing research has generally focused on an association between religiousness and health, addressing both prayer (Krause, Chatters, Meltzer, \& Morgan, 2000) and attendance at religious services (McCollough, Hoyt, Larson, Koenig, \& Thoresen, 2000).

While these studies suggest that continued involvement with religious activities is an important factor in maintaining good health throughout life, they do not discuss the importance of religiousness and spirituality in the everyday decision-making process. It is important to address religiousness and spirituality in the problem-solving and decision-making process because most individuals feel a strong connection with religion and/or spirituality. Results from the most recent Gallup Poll (2005) show that a majority $(80 \%)$ of the individuals polled are convinced that God exists and even more $(90 \%)$ have a religious affiliation. In addition, many of these individuals (65\%) are members of a church or synagogue and most (58\%) attend services on a regular basis. Even more surprising is the fact that over half $(58 \%)$ of those polled believe that religion can answer most of today's problems. Therefore individuals may make decisions or select strategies based on their religious beliefs or spirituality. Thus, religion and spirituality should not be 
ignored as influences in daily decisions and need to be included in problem-solving and decision-making research.

One exception to the lack of religiousness and spirituality in research are those studies examining highly emotional end-of-life decisions. Although limited, research in the area of end-of-life decisions has also employed hypothetical vignettes. The vignettes generally feature an individual in a terminal or chronic health condition facing a treatment or suicide decision. Similar to other decision-making studies, rather than addressing strategies, the focus is typically on the final end-of-life solution to the problem presented. Results of these studies suggest that age, gender, self-assessed health, and religiousness influence the types of end-of-life decisions endorsed in the hypothetical situations (Cicirelli, 1997a, 1997b, 1998; Cicirelli, MacLean, \& Cox, 2000; Mills \& Wilmoth, 2002; \& Wellman \& Sugarman, 1999). However, no single study has addressed all five of these factors concurrently in relation to end-of-life decisions. Moreover, although age, gender, health and religiousness have been considered in these investigations, spirituality has not been examined. Thus, the literature examining the factors that influence end-of-life decisions has overlooked the complex interplay among these factors.

Another limitation to the scope of this literature focuses on the fact that researchers have rarely examined both strategy and decisional outcome concurrently. One exception is Patrick \& Strough (2004), who used hypothetical vignettes to examine the relocation intentions of older adults. Participants were asked to generate strategies to solve the hypothetical vignettes as well as respond to a question regarding their own personal intentions of relocating. Results of this study demonstrated that the measures 
obtained in the lab related to the strategies used for the hypothetical vignettes, and also to intended real world behavior. Building on the logic of Patrick and Strough (2004), the current study examined the associations of five predictors (age, gender, religiousness, spirituality, and self-assessed health) in relation to the strategies and decisional outcomes generated in response to three different decisional domains: a lowemotionally salient consumer decision, a medium-emotionally salient family decision, and a high-emotionally salient end-of-life decision. Such an examination may provide answers to questions regarding whether age differences in strategy are related to the observed differences in decisional outcome and whether religiousness and spirituality are important in multiple decisional domains.

To facilitate our understanding of the decision-making process then, it is necessary to examine when age, gender, religiousness, spirituality, and self-assessed health do, and do not, influence the decision-making process. Thus, the purpose of the current study was: 1) to examine the influence of age, gender, religiousness, spirituality, and self-assessed health simultaneously as they relate to three decisional domains, but specifically to the end-of-life decisional domain; 2) to investigate the pervasiveness of religiousness and spirituality across three different decisional domains (consumer, family, and end-of-life), each of which elicits a different level of emotional salience (low, medium, and high); and 3) to examine the link between problem-solving strategies and decision-making outcomes. 
Chapter 2: Introduction

Making decisions is an integral part of everyday life for all individuals (Yates \& Patalano, 1999). Some decisions involve little emotion and are made relatively frequently, including those involving meals, clothing choices, or vacations (BlanchardFields, 1998). Other decisions are highly emotional and relatively rare, such as those related to health (Willis et al., 1999), relocation (Patrick \& Strough, 2004), and end-oflife (Cicirelli, 1997a). Studies examining everyday decisions generally focus on either the strategies used in the process of solving the problem or the actual decisions made in response to the problem situation.

\section{Problem-Solving Strategies}

Research in the area of problem solving was developed to aid in understanding how adults solve realistic problems. Difficulties arise, however, when trying to equate the problems addressed in the lab to problems that occur in real life. Reese (1992) defined and described two types of problems. Well-defined problems contain components that are laid out clearly enough for the individual to see, and the outcome involves a single solution or a clearly defined goal (Allaire \& Marsiske, 2002; Sinnott, 1998). In contrast, ill-defined problems often have indeterminate goals and more than one acceptable solution, making them difficult to solve (Allaire \& Marsiske, 2002; Marsiske \& Willis, 1995; Reese, 1992; Sinnott, 1998). Thus, ill-defined problems are more similar than well-defined problems to situations that are seen in everyday life, although the everyday problem-solving experience actually involves a combination of both types of problems (Sinnott, 1998). 
Researchers examining everyday problem solving, and the factors that influence an adult's problem-solving process, often use hypothetical vignettes to represent a realistic situation (Blanchard-Fields et al., 1995; Blanchard-Fields, Chen, \& Norris, 1997; Cornelius \& Caspi, 1987; Denney \& Pearce, 1989; Patrick \& Strough, 2004). Participants are asked to read the vignettes and indicate what they would do, or what the target person should do, to solve each of the hypothetical problems. Past research on everyday problem solving has focused on either the number of strategies generated by the participants to solve each situation (Berg et al., 1999; Denney \& Pearce, 1989) or the type of strategies given in response to the various hypothetical situations (Blanchard-Fields et al., 1995; Cornelius \& Caspi, 1987).

Number of strategies

Age differences have been reported in the number of strategies offered in response to hypothetical situations. For example, Denney and Pearce (1989) found that middle-aged adults offered more strategies than both younger and older adults. In contrast, Berg et al. (1999) found that younger adults generated more problem-solving strategies than older adults. Although Patrick and Strough (2004) did not specifically examine age differences, they found that experience with a particular problem related to more total strategies generated in response to the problem vignettes. Although the results of these studies are valuable, focusing solely on the number of strategies generated leaves an incomplete picture of the problem-solving process. For this reason, Patrick and Strough (2004) recommend that researchers also examine the type of strategies generated. 
Type of strategies

Studies investigating the type of strategies offered in response to hypothetical vignettes either asked the participant to generate their own strategies (Blanchard-Fields et al., 1995; Denney \& Pearce, 1989) or to choose strategies from those provided (Cicirelli, 1997a; Cornelius \& Caspi, 1987; Watson \& Blanchard-Fields, 1998). The strategies given in response to the vignettes are then coded for specific types. One common way to code strategies includes whether the participant chose to focus specifically on changing the problem—problem-focused strategies—or to focus on changing the emotions they feel because of the problem—emotion-focused strategies (Blanchard-Fields et al., 1995). Although mixed, age differences in the use of problemfocused and emotion-focused strategies have been reported (Blanchard-Fields et al., 1995; Strough, Berg, \& Sansone, 1996). For example, Blanchard-Fields et al. (1995) found that younger adults used more problem-focused strategies than the older adults while Blanchard-Fields et al. (1997) found that problem-focused strategies were preferred by all participants regardless of age. In contrast, Cornelius and Caspi (1987) reported no age differences in the type of strategies chosen, with the exception that the older adults suggested turning to religion more often than the younger and middle-aged adults. Thus the evidence on age differences in problem-solving strategies is equivocal and needs to be examined further.

Strategy type may vary as a function of whether the individual feels that the problem or its solution involves the self, others, or the environment (Berg, Strough, Calderone, Sansone, \& Weir, 1998). The problem domain influences strategy type (Blanchard-Fields et al., 1995; Cornelius \& Caspi, 1987; Watson \& Blanchard-Fields, 
1998). For example, when specific domains are examined—such as school, friends, and leisure (Berg et al., 1998); consumer, work, health, family, and caregiving (Blanchard-Fields et al., 1995); and relocation (Patrick \& Strough, 2004)—participants tend to endorse changing themselves in order to solve the hypothetical situations presented more often than any other type of strategy.

\section{Decision-Making Outcomes}

In contrast to problem-solving studies that address the number or type of strategies used to solve the problem, decision-making studies focus on the outcomes, or final decisions made in response to the problem. Because the goal of decisionmaking is to determine a satisfying solution to the problem, decision-making may be viewed as one kind of problem-solving (Yates \& Patalano, 1999). Just as individuals generate different numbers or types of strategies, individual decisions also vary depending on the characteristics of the individual or the domain of the problem-solving situation (Cicirelli, 1997a).

One of the main objectives of decision-making studies has been to find a link between decisions made in the laboratory and those made in real-life situations. Meyer, Russo, and Talbot (1995) conducted two studies—one addressing decision-making in the lab and the other addressing decisions made in real-life. Study one used hypothetical vignettes to represent a situation in which an individual was diagnosed with breast cancer. After reading the hypothetical situation, participants were asked to make a decision regarding treatment options and then explain the reasoning behind their choice. The authors then conducted study two, consisting of an anonymous survey to women recovering from breast cancer, which allowed for further investigation of the 
relation between the decisions made in the lab and those made in real-life. Results from these two studies showed an age difference in decision-making, with older women seeking less information than younger women before deciding to seek treatment. More importantly, the results also showed that the treatment decisions made in response to the hypothetical vignette in study one related to the real-life decisions made by the participants surveyed in study two (Meyer et al., 1995).

Diehl, Willis, and Schaie (1995) also addressed the link between lab measures and real-life decisions with a study on instrumental activities of daily living (IADLs). In addition to the paper and pencil measures conducted in the lab, the authors included a home visit in which the participants were observed performing IADLs in three different domains-preparing food, taking medications, and answering the phone. Results of this study showed convergent validity between the two measures revealing that the measures completed in the lab correlated to the real-life behaviors observed in the home. The results also suggested that there was more than one way to correctly perform the IADLs—-the choices made by one participant may not have worked for another individual in the same situation (Diehl et al., 1995). These differences suggest that it is important to continue with an examination of the link between problem-solving strategies and decision-making outcomes.

\section{Decision-Making Processes and Outcomes}

Linking decisions made in the lab to everyday decisions is important. However it is also important for researchers to focus on the decision-making process and to establish a link between strategies generated in response to a problem situation and the final decision made to solve the problem. This link is important because researchers 
investigating only strategies, or only outcomes, are not focusing on an actual understanding of the overall decision-making process, but rather on specific individual differences in strategies or outcomes. For example, past research has shown that the strategies generated in response to a problem-solving situation differ as a function of both the domain of the problem and the age of the participant (Blanchard-Fields et al., 1995). Past research has also shown that, when examining an end-of-life domain, outcomes such as maintain the life versus end the life differ as a function of individual characteristics such as age, gender, and religiousness; and different problem domains such as terminal versus chronic illnesses (Cicirelli, 1997a). Therefore, without addressing strategies and outcomes concurrently, researchers will continue to catalog individual differences in one specific aspect of decision-making rather than attempting to understand how to improve the overall decision-making process.

One exception, to the existing literature focusing on strategies versus outcomes, is Patrick and Strough (2004) who examined the relation between relocation strategies and decisional outcomes. The goal of this study was to link the strategies generated in response to hypothetical vignettes in the lab to intended real-world behavior. The vignettes provided a rare, high emotional situation often faced in late life, that of relocation. The participants generated strategies in response to the vignettes and answered a question concerning intentions of relocating in their own life. Findings indicated that not only is there a link between the decisions made in the lab and the participants' intended real-life decisions via individual differences in experience, but there is also a link between the strategies generated in response to the vignettes and the intended decision that may ultimately be made. Thus, in this study, differences in 
strategies were related to observed differences in decisional outcomes (Patrick \& Strough, 2004).

\section{Emotional Salience}

According to Blanchard-Fields (1998), emotion is an integral part of an individual's decision-making process and shares a reciprocal relation with the cognitive processes used in learning and decision-making. Recently, researchers began to address the role that emotion plays in the problem-solving process as well as in decision-making outcomes. The level of emotion that is involved in, or elicited from, a particular problem has been termed emotional salience (Blanchard-Fields, 1986). Blanchard-Fields et al. (1995) used open-ended hypothetical vignettes that had been rated in a pilot study as eliciting low-, medium-, or high-emotional salience. Participants were asked to read the vignettes, write out their solutions to the problems, and rate the vignettes for level of emotional salience. Results of this study suggested that the level of emotional salience elicited by the hypothetical vignette was associated with both the number and type of problem-solving strategies endorsed to solve the problem. Specifically, as the level of emotional salience increased, the number of emotionfocused strategies generated in response to the vignette also increased, while the number of problem-focused strategies decreased (Blanchard-Fields et al., 1995).

In a slightly different study addressing emotional states, Blanchard-Fields, Chen, Schocke, and Hertzog (1998) used twelve open-ended vignettes and asked their participants to rate the level of emotion elicited from each of the situations and to explain how familiar they were with each problem. Results suggested that, when replying to the vignettes, the participants made their decisions based on their own 
personal beliefs and/or values. Therefore, the domain of the problem situation evokes not only emotion, but also attitudes, beliefs, and values, which in turn influence the strategy or decision chosen in response to the problem (Blanchard-Fields et al., 1998). Thus, emotion is an essential component in the decision-making process, mirroring Blanchard-Fields $(1986 ; 1998)$ original thoughts on the role of emotional salience in the decision-making process.

\section{Religiousness and Spirituality}

In order to study religiousness and spirituality, researchers have adopted the standard practice of differentiating, and even completely separating, these two concepts (Emmons \& Paloutzian, 2003; Hill \& Pargament, 2003; Pargament, 1992; Pargament, 2000; Wink \& Dillon, 2003; Zinnbauer, Pargament, \& Scott, 1999). In general, religiousness encourages moral standing and participation in activities that attest to a belief in God or a higher power (Emmons \& Paloutzian, 2003). Therefore, religiousness is represented as a narrow construct that encompasses an outward expression of organized behaviors and commitments such as church attendance (Hill \& Pargament, 2003). Spirituality, on the other hand, refers to a range of experiences that embrace the existence of God or a higher power, but without requiring the actual practice of, or participation in, organized religious activities (Emmons \& Paloutzian, 2003). This definition leads to a representation of spirituality as being a more personal, subjective, and inward emotional experience without the public expression of religious behaviors (Hill \& Pargament, 2003).

To further examine these concepts, Zinnbauer, Pargament, Cole, Rye, Butter, Belavich et al. (1997) conducted a study to assess how individuals conceptualize 
religiousness and spirituality. The participants indicated their own level of religiousness and spirituality in addition to writing definitions for each and describing how they relate to each other. Results of this study showed that both religiousness and spirituality have certain aspects in common, specifically church attendance and the amount of time spent in prayer. In addition, the definitions of both religiousness and spirituality given by the participants included references to time-honored concepts surrounding religionthose of God and the church—showing once again that the two concepts are related (Zinnbauer et al., 1997). However, a study conducted for the American Association of Retired Persons (AARP; Montenegro, 2004) showed that religiousness and spirituality, though related, are not synonymous. Individuals 45 years of age and older were polled as to their level of religiousness and spirituality. Of the individuals responding to the survey, $80 \%$ rated themselves as very religious and $85 \%$ rated themselves as very spiritual. To further investigate the relation of religiousness and spirituality, those individuals who had rated themselves as being very religious were asked if they also considered themselves to be spiritual and vice versa. In response to this question, $81 \%$ of those who were religious also rated themselves as being spiritual but only $66 \%$ of those who were spiritual rated themselves as being religious. Thus, in the current literature, religiousness and spirituality continue to be conceptualized as two separate, but related, constructs.

Although investigations of problem-solving and decision-making have begun to take the influence of emotions into account, the relevance of religiousness and spirituality to strategies and outcomes is still understudied. For example, research studies that do address the relation of religiousness and/or spirituality to decision- 
making tend to focus on social behavior. Bonner, Koven, and Patrick (2003), examined depressed affect and prosocial behaviors using religiousness and spirituality as predictors. Results of this study suggested that both spirituality and religiousness emerged as significant predictors of prosocial behavior, with spirituality having a greater influence than religiousness on the helpful solutions offered in response to hypothetical vignettes. Another area of research receiving increased empirical attention includes the ways in which religiousness and spirituality influence coping behavior. Researchers addressing religious coping have found that both religiousness and spirituality are important factors in coping with a crisis or other critical life event (Harrison, Koenig, Hays, Eme-Akwari, \& Pargament, 2001).

Although these results are informative, and show the importance of both religiousness and spirituality in different areas of research, very little is known concerning the influence of these predictors in the decision-making process. A limited amount of research has found that religion is important in problem-solving, and that many individuals turn toward religion and spirituality when engaging in the problemsolving process. For example, Pargament, Kennell, Hathaway, Grevengoed, Newman, and Jones (1988) found that religion influences problem-solving through three different styles of coping. In addition, Cornelius and Caspi (1987) found that the older adults suggested turning to religion as a problem-solving strategy more often than younger and middle-aged adults. The findings that older adults are more likely to offer religion as a coping or problem-solving strategy may be a function of the domains investigated. Thus, the pervasiveness of religiousness and spirituality across multiple domains of decision-making with differing levels of emotional salience is still virtually unknown. 


\section{Hypothetical End-of-Life Decisions}

One area of decision-making that integrates emotional salience and religiousness is the examination of end-of-life decisions. Similar to problem-solving and decision-making studies, research in the area of end-of-life decisions often employs hypothetical vignettes (Cicirelli, 1997a, 1997b, 1998; Mills \& Wilmoth, 2002; Wellman \& Sugarman, 1999). For example, Mills and Wilmoth (2002) used a cross-sectional sample of 3-generation families to examine attitudes regarding hypothetical end-of-life decisions, specifically the right to die and the right of family members to make proxy end-of-life decisions. Results of this study showed no significant age differences in attitudes toward the specific end-of-life decisions examined. However, there were significant differences in religiousness. For example, a higher level of religiousness was associated with a lower endorsement of both believing in the individual's right to die and believing in the right to make proxy end-of-life decisions for family members who can no longer make those decisions on their own.

Wellman and Sugarman (1999) also addressed end-of-life decisions but looked specifically for age differences between younger and older adults. To address the sensitive issue of withdrawal from medical treatment, participants were each given a hypothetical vignette about a middle-aged man who was critically ill. He was admitted to the hospital during a medical emergency and later died. Although each participant received the same vignette, the conditions under which the man died differed including: take all measures to extend the life, take no measures to extend the life (i.e., do not resuscitate), withdraw treatment, or use of a lethal injection to commit suicide. Results 
of this study revealed that younger and older adults had comparable attitudes toward accepting the patient's refusal to continue medical treatment when death was imminent.

In a series of reports, Cicirelli (1997a; 1997b; 1998), examined the associations among age, gender, self-assessed health, religiousness, and end-of-life decisions. Older adults were presented with seventeen hypothetical vignettes featuring individuals in one of three domains: terminal physical health conditions, chronic physical health conditions, and mental health conditions. After reading the vignette, participants were asked to indicate their likelihood of engaging in each of seven end-of-life decision options. A factor analysis narrowed the end-of-life decision options to three: maintain the life, defer the decision to someone else, or end the life. Age differences in decision outcomes emerged, with the old-old (ages 75 to 84 years) and the oldest-old (ages 85 to 100 years) groups being less likely to endorse the decision to maintain life than the young-old group (ages 60 to 74 years). Instead, the adults over the age of 75 endorsed the decision to defer to someone else (Cicirelli, 1997a). In addition, gender differences emerged; men were more likely to favor ending the life, while women were more likely to endorse maintaining the life or refusing medical treatment and allowing the life to end naturally. No differences in endorsement of specific end-of-life decisions were found in relation to the self-assessed health of the participants (Cicirelli, 1997a; 1998).

The overall results of this series of reports show a strong, consistent relation between religiousness and end-of-life decisions in that those who profess to have stronger religious beliefs tend to favor maintaining the life rather than ending it. In fact, the importance of religion, God, and prayer in the respondent's life were consistently related to a preference for maintaining the life, showing that religiousness matters in 
high-emotionally salient end-of-life decisions (Cicirelli, 1997a; 1997b; 1998; Cicirelli et al., 2000). Although important, there are limitations to this research. These reports examined the associations of age, gender, religiousness, and self-assessed health specifically in relation to end-of-life decisions, ignoring other decisional domains and ignoring the influence of spirituality. In addition, the respondents were limited to older adults, 60-100 years of age. The close-ended response format may have limited the range of options considered. Moreover, the strategies used to solve the problem were not addressed in the close-ended format. Thus, further investigation is warranted.

\section{Summary of Current Study}

In order to advance this area of investigation, it is necessary to examine when these constructs do, and do not, influence the overall problem-solving and decisionmaking process. To account for the aforementioned limitations, therefore, the current study used two different age groups-younger adults (18-25 years of age) and older adults (55-84 years of age). In addition, the current investigation examined the associations of five predictive factors (age, gender, religiousness, spirituality, and selfassessed health) as they related specifically to the high-emotionally salient end-of-life decisional domain. Participants were asked to write their own responses to hypothetical open-ended vignettes rather than endorsing forced-choice answers. In order to address the link between the strategies and decisional outcomes, the outcomes for the end-oflife vignette in the current study were generated from the total written response given in solving the hypothetical end-of-life situation. Finally, the investigation of strategies across the three domains addressed differences in strategies generated in each domain and each level of emotional salience. 
Although the current study addressed the limitations in the prior literature and attempted to address multiple predictors in the decision-making process, the crosssectional design of the study also poses concerns. For example, the use of only two age groups is not inclusive and the results obtained may have more to do with cohort differences rather than specific age differences. In addition, not all of the possible predictors of everyday problem solving and end-of-life decision-making were included in the study. A "third variable problem" may exist in that the variables used to predict the problem-solving strategies and end-of-life outcomes may be related through a variable that was not included in the study. For example, religiousness and spirituality may be related to end-of-life decisional outcomes because individuals of a specific personality type tend to be more religious or more spiritual than those individuals with a different personality type. However, the current study does offer an initial examination of the variables involved in everyday problem-solving and end-of-life decision-making and the potential roles of religiousness and spirituality. Although the design is not perfect, the results add important information to the existing literature.

Thus, the current investigation examined the influence of age, gender, religiousness, spirituality, and self-assessed health simultaneously as they related specifically to the high-emotionally salient end-of-life decisional domain; investigated the limits of religiousness and spirituality across three different decision domains (consumer, family, and end-of-life), each with a different level of emotional salience (low, medium, and high); and examined problem-solving strategies and decisional outcomes concurrently. In order to address these issues, the current investigation was guided by the following research questions and hypotheses: 


\section{Research Questions and Hypotheses}

\section{Decisional Outcomes}

Research Question 1: What are the associations among age, gender, religiousness, spirituality, and self-assessed health in relation to end-of-life decisions?

Hypothesis 1: Age: No age differences between younger and older adults are expected in the type of end-of-life decision that is made (Wellman \& Sugarman, 1999; Mills \& Wilmoth, 2002).

Hypothesis 2: Acceptance for specific end-of-life decisions will differ as a function of individual characteristics other than age. Specifically,

a. Gender: Men are expected to favor the decisions of suicide or assisted suicide while women are expected to accept death, do all they can to fight against death, or defer the decision to others (Cicirelli, 1997a).

b. Religiousness: Because individuals with a higher level of religiousness can be expected to endorse dogmatic precepts admonishing suicide, they are expected to accept death or defer their decision to others.

c. Spirituality: Because religiousness and spirituality have been shown to be overlapping constructs (Zinnbauer et al., 1997), individuals who report a higher level of spirituality are expected to accept death or defer their decision to others, while those with a lower level of spirituality are expected to do all they can to fight the prognosis of death or give up and choose suicide or assisted suicide.

d. Self-Assessed Health: Despite Cicirelli's (1997a) null finding between endof-life decisions and self-assessed health, it is expected that, when 
addressing differences between younger and older adults, those who report lower self-assessed health will choose to accept death, choose suicide, or endorse assisted suicide. In contrast, those with higher selfassessed health are expected to endorse fighting the prognosis or to defer to others for a decision.

Hypothesis 3: Religiousness versus Spirituality: Because religiousness is seen as a narrow construct that encompasses an outward expression of a professed commitment to God, or a higher power, (Hill \& Pargament, 2003) organizational religiousness may limit the range of options an individual has in regard to end-of-life decisions. Therefore, spirituality is expected to have a greater effect on individual endorsement of end-of-life decisions over the additional factors of age, gender, health, and religiousness.

\section{Problem-Solving Strategies}

Research Question 2: Is the influence of religiousness and spirituality pervasive across decisional domains with differing levels of emotional salience or only influential in highly emotional decisions?

Hypothesis 4: Due to known variation in the strategies employed in response to differing levels of emotional salience (Blanchard-Fields et al, 1995) and Cicirelli's (1997a, 1997b, 1998) findings that religiousness is important in end-of-life decisions, religiousness and spirituality are not expected to affect the strategies used in response to the low-emotionally salient consumer decision or the mediumemotionally salient family decision, but will relate to the strategies offered in response to the high-emotionally salient end-of-life decision. 


\section{Exploratory Research Question}

Research Question 3: Is there a link between the type of strategies generated in response to the end-of-life decisional domain and the participants' final decisional outcomes? If so, what does that link add to the current fields of problem-solving and decision-making research? 


\section{Chapter 3: Methods}

\section{Participants}

Data for the current study were provided by 187 adults. In order to allow for comparative analyses, the participants were divided by age into two groups consisting of younger and older adults. Nine participants were eliminated from the current sample to allow for a clearer distinction of the two age groups. Thus, the final sample consisted of 100 younger adults and 78 older adults.

Younger Adults

One hundred participants (55\% women) with an age range of 18 to 25 years ( $M$ $=19.9, S D=1.5)$ provided the data for the group identified as younger adults.

Recruitment was accomplished through a posted flyer on the Psychology Department bulletin board at West Virginia University. Participants were enrolled in undergraduate psychology courses at the time of the study and received extra credit points for the time needed to complete the survey. The younger adults represented the Mid-Atlantic States of West Virginia, New Jersey, and Maryland with one younger adult each representing Virginia and North Carolina.

\section{Older Adults}

Seventy-eight participants (64.1\% women) with an age range of 55 to 84 years $(M=71.1, S D=7.9)$ provided the data for the group identified as older adults. The data for this group were the result of a combined data set consisting of existing data collected in 2002 and new data collected in $2004^{1}$. The existing data were provided by a

\footnotetext{
${ }^{1}$ Exploratory independent samples $t$ tests comparing the 2002 and 2004 samples revealed no significant group differences on the variables of interest to this study. Thus, the two samples were combined for the current analyses.
} 
subset of 58 older adults who completed an extensive battery of cognitive and decisionmaking tasks and self-administered surveys during the summer of 2002 (R03 AG19917). These participants resided in the Mid-Atlantic States (i.e., West Virginia, Ohio, and Pennsylvania) and were recruited through published ads in local newspapers, flyers posted in community centers, and word-of-mouth referrals. Each participant received a cash honorarium upon completion of the protocol. Additional data collection took place during the fall of 2004 . The new data were provided by a sample of older adults residing in the same Mid-Atlantic States of West Virginia, Pennsylvania, and Ohio. Potential participants received a packet in the mail consisting of a cover letter, survey booklet, and informed consent forms. Of the 250 older adults who received the packets, 30 completed surveys and consent forms were returned and used in the current study.

The final sample for the current study then, included 45 younger men, 55 younger women, 28 older men, and 50 older women for a total of 178 participants (see Table 1 for frequencies of total sample). Power analyses, implemented in Sample Power, suggested that a sample size of 140 would provide sufficient power (power > $.80)$ to detect medium-sized effects $(f=.25)$ at $p<.05$ for mean comparisons between two groups and medium-sized effects $(f=.25)$ at $p<.05$ for mean comparisons among four groups. In addition, a sample of 120 would provide sufficient power (power $>.80$ ) to detect medium-sized effects $\left(f^{2}=.15\right)$ using up to 10 predictors in a multiple linear regression analysis. Therefore, the sample size in the current study provides sufficient statistical power to test the hypotheses of interest. 


\section{Procedure}

The procedures in the current study included an anonymous, self-administered survey completed individually or in small groups of $5-10$. All participants were instructed as to the purpose of the study and gave their consent to participate. Data collection for the younger adults took place in the Psychology Department of West Virginia University. Participants came to a designated classroom, read and signed the informed consent form (see Appendix A) and completed the survey booklet. The surveys were completed without assistance and participants were given as much time as needed to answer the questions. Data collection for the older adults included a selfadministered survey packet received though the mail. The survey packets included a cover letter (see Appendix B), informed consent form (see Appendix C), and the survey booklet. Each participant read and signed the informed consent form and mailed it back in the return envelope when the survey booklet was complete.

\section{Measures}

\section{Decision Vignettes}

Six different hypothetical vignettes were presented to the participants, three of which were used in the current study ${ }^{2}$. Two of the vignettes characterized decisions that are common and tend to be made in everyday life, while the third vignette corresponded to a rare end-of-life decision (see Appendix $D$ for presentation of full vignettes). The first vignette, designed by Patrick (2000) for use in an earlier study, represented an end-oflife decision. The individual featured in this vignette has been told by his doctor that he has less than one year to live. His remaining months are likely to be very painful and he

\footnotetext{
${ }^{2}$ The participants in the 2002 data collection responded to ten vignettes, three of which were the same as the vignettes used in the current study.
} 
is thinking about committing suicide. The second vignette, taken from the work of Blanchard-Fields et al. (1995), represents a medium-emotionally salient family decision. The individual featured in this vignette has an 86-year-old grandmother who still enjoys driving and feeling independent. However, the individual feels that the grandmother is no longer capable of driving safely and that her driving is a danger to herself and the community. The third vignette was also taken from the work of Blanchard-Fields et al. (1995), and represents a low-emotionally salient consumer decision. The individual featured in this vignette has bought some merchandise for her daughter that is clearly defective. She tries to return the merchandise, but the store manager refuses to take it back. To elicit responses to the problem situations presented in the vignettes, the participants were asked one open-ended question, "What should the person do?" No instructions were offered as to how many, or what type of, responses to give, only that they should read the vignettes and write their own answers to the question.

The two vignettes from Blanchard-Fields et al. (1995) were shown to be low- and medium-emotionally salient through ratings obtained from the participants in that study; however, the end-of-life vignette was untested for emotional salience. Therefore, to determine the level of emotional salience elicited by each of the vignettes, a pilot study was conducted (see Appendix E). The three vignettes used in the current study were presented to both older and younger community dwelling adults $(N=8)$. Through the ratings received from the pilot study, each vignette was determined to represent a different level of emotional salience. For this pilot study, Kendall's coefficient of concordance showed perfect agreement on salience ratings across the three vignettes used in the current study $\left(W=1.0 ; X^{2}(2, N=8)=16.0, p<.001\right)$. In terms of level of 
emotional salience for the current study, the consumer vignette was of low emotional salience, the family vignette represented medium-emotional salience, and the end-of-life vignette was of high emotional salience.

\section{Demographics Background}

The survey packet included a general demographics questionnaire assessing several self-reported measures including chronological age, marital status, religious affiliation (if any), race, gender, education level, household income, and a question assessing difficulty paying bills. Each of these was assessed through the use of a single question as shown in Appendix F. Table 1 presents a summary of the descriptive characteristics and frequencies for the total sample $(n=178)$ as well as the division between younger and older adults.

\section{Self-Assessed Health}

In addition to the demographic variables, the participants were asked to assess their current overall health. Four questions of self-reported health from the Multilevel Assessment Instrument were included in the demographics section of the questionnaire (Lawton, Moss, Fulcomer, \& Kleban, 1982; see Appendix G). The first question asked the participants to rate their own overall health. Responses to this question were rated on a 4-point Likert scale from 1 (poor) to 4 (excellent). Two of the following questions focused on comparing the participant's current health to their past health and to other individuals the same age. The responses were rated on a 3-point scale from 1 (not as good) to 3 (better). The remaining question asked whether the participant's current health issues prevented them from doing what they want to do. Reponses were rated once again on a 3-point scale from 1 (a great deal) to 3 (not at all). Scoring range for 
these four questions is $4-13$ with a higher score indicating better overall self-assessed health. The actual range of scores for the participants in the current study was $6-11$ ( $M$ $=9.1, S D=1.2$; see Table 2). The internal consistency of this 4-item multidimensional scale was low, with a Cronbach's alpha of .66.

Religiousness

Organizational religiousness was measured with a 4-item scale taken from the work of Kenney, Vaughn, and Cromwell (1977). This scale addressed the frequency (1 $=$ never to $4=$ very often) of participating in various religious activities including attendance at religious services and/or religious meetings, monetary contributions, and membership in religious organizations (see Appendix $\mathrm{H}$ ). Scores for this scale can range from 4 to 16 with higher scores indicating higher organizational religiousness. The actual scoring range for this sample was $4-16(M=10.8, S D=3.9$; see Table 2$)$. A Cronbach's alpha of .92 in this sample indicates high internal consistency among the scale items.

Spirituality

Spirituality was assessed using the Universality subscale of the Spiritual Transcendence Scale (Piedmont, 1999). The Spiritual Transcendence Scale was used to assess the participant's level of spirituality because it defines spirituality as transcendental, containing multidimensional facets different from organizational religiousness. The Universality subscale is a 9-item self-report scale that assesses spirituality through belief in a meaningful purpose in life (see Appendix I). Responses on this subscale were rated on a 5 -point Likert scale from 1 (strongly disagree) to 5 (strongly agree). Scoring range on the subscale is $9-45$ with higher scores indicating 
higher spirituality. The actual scoring range for this sample was $15-44(M=34.1, S D=$ 5.3; see Table 2). This subscale demonstrated acceptable internal consistency with a Cronbach's alpha of .80 .

\section{Coding Scheme}

The written responses from the three decision vignettes were assessed and coded for strategies given to help the individual in the vignette to solve the problem. Reliability of coding was established between the principal investigator and her faculty advisor using two measures of inter-rater reliability—Kappa coefficients and Intra-class correlations. Kappa coefficients $(\mathrm{K})$ were used in this study because they determine inter-rater reliability by focusing specifically on the agreement between coders (Howell, 2002). Kappa coefficients were computed on the overall coding of the problem-solving strategies, the overall coding of the decisional outcomes, and on the coding of each individual strategy. Intra-class correlations (ICC) also address agreement between coders. However, these correlations also take the disagreement between coders into account offering a more reliable measure of agreement (Howell, 2002). Thus, intra-class correlations were computed to ensure inter-rater reliability between the coders, especially on those individual strategies with lower Kappa coefficients.

To determine inter-rater reliability in the current investigation, the two coders individually coded $21 \%$ of the data and together reached reliability with a Kappa coefficient of .81 and an intra-class correlation of .90 on the problem-solving strategies. The coders reached perfect reliability on the decisional outcomes with a Kappa of 1.00 (see Appendix $\mathrm{J}$ for the coding sheet used in this study). Kappa coefficients for interrater reliability on the individual strategies ranged from .67 to .98 ; and intra-class 
correlations ranged from .81 to .99 . Once inter-rater reliability was reached, the remaining surveys were completed by the principal investigator. Responses to all three vignettes were coded for both number and type of problem-solving strategies, while the end-of-life vignette was also coded for decisional outcomes. Coding all three vignettes in the same way made it possible to compare the type of strategies generated in response to the varying levels of emotional salience across the three decisional domains. Decisional outcomes were only coded for the end-of-life domain; therefore, it was not possible to directly compare outcomes across the decisional domains.

\section{Problem-Solving Strategies}

Strategies suggest possible solutions in dealing with the hypothetical situation presented. Each specific idea or thought suggested by the respondents was coded as a separate strategy. Therefore, multiple strategy codes were given if the respondent suggested more than one strategy, or more than one suggestion was offered of the same strategy type. The responses to all three hypothetical vignettes were coded for type of strategies using a combination of existing coding schemes (Berg et al., 1998; Strough, Patrick, \& Swenson, 2003; Swenson, 2003; Patrick \& Strough, 2004). The coding scheme developed by Berg et al. (1998) reduces open-ended responses to four different types of problem-solving strategies including: regulate own behavior, regulate own cognition, change the environment, and change other behavior. From this coding scheme, five coding strategies were developed—change own cognition, change own behavior, change other behavior, change the environment and change to a new environment. In addition to these five strategies, eight additional strategy types, taken from Patrick and Strough (2004) and Strough, Patrick, and Swenson (2003), were 
coded: change own emotion, change other emotion, change other cognition, cooperate/ discuss, seek information, seek social support, and seek formal support. The final strategy type to be coded was religion and was used in Strough et al. (2003) although it was not endorsed by any of the participants in their study of grandparenting decisions. In addition to informing the types of strategies to be coded in this study, Swenson (2003) provided a model for compiling the thirteen strategies into a coherent system (see Appendix $\mathrm{K}$ for the coding manual used for the strategies). The thirteen strategies coded in the current study are detailed below.

Cooperate/discuss and seek information are strategies aimed at involving other individuals in the decision, discussing options, or actually asking others for information that could be used to solve the problem. Examples of these strategies include seeking a second opinion or involving the family in the decision process. Because there were multiple, discrete behavioral responses suggested with each of these strategies, whenever a coder designated a strategy as cooperate/discuss or seek information, it was also coded as the most specific behavioral strategy (e.g., change own behavior; seek social support; seek formal support). For this reason, the strategies of cooperate/discuss and seek information were not included in the final analyses. However, they were included in computing Kappa coefficients for each individual strategy. The agreement between the coders for these strategies was .90 for the strategy of cooperate/discuss and .68 for seek information (Intra-class correlations $=.95$ and .81 , respectively).

Strategies aimed at changing and/or regulating the self included suggestions that the actor in the vignette could do on their own to solve the problem. For example, 
change own emotion included suggestions such as thinking positively, looking for the good in the situation, and trying to enjoy life. The strategy of change own cognition was coded if the respondent suggested a change in thought such as realizing that he or she is not a burden to her family or reconsidering the grandmother's ability to drive. The strategy of change own behavior included any suggestion that the individual in the vignette take personal responsibility for solving the problem. Examples included volunteering in the community to combat loneliness, shopping at a different store to solve the consumer problem, or volunteering to drive to solve the problem of the grandmother's driving ability. The Kappa coefficients for these strategies were .81 for change own emotion, .77 for change own cognition, and .85 for change own behavior (Intra-class correlations $=.89, .87$, and .92 , respectively).

Strategies aimed at changing others focused once again on emotional, cognitive, and behavioral change. For example, change other's emotion included suggestions such as helping the grandmother to feel needed, and making sure the store manager felt guilty about not taking back the dresses. The strategy of change other's cognition included assisting the individual's family to understand the end-of-life decision that has been made, or convincing the grandmother that she no longer needs to drive and helping her to understand that she may be putting others in danger by continuing to drive. The strategy of change other's behavior included convincing other people to take their business to another store or teaching the grandmother to take the bus. The Kappa coefficients were .67 for change other's emotion, .77 for change other's cognition, and .74 for change other's behavior (Intra-class correlations $=.80, .87$, and .85 , respectively). 
Some strategies involved seeking support from others. Seek social support included strategies asking family or friends for assistance; while those strategies asking doctors or other professionals for advice or support were coded as seek formal support. One other strategy that included the idea of support was religion. Religious strategies included all responses suggesting that the individual turn toward religion such as pray, talk with a pastor, or make things right with God. Agreement between coders for these strategies was high, with the Kappa equal to .94 for seek social support, .90 for seek formal support, and .98 for religion (Intra-class correlations $=.97, .95$, and .99 , respectively).

Strategies generated could also involve the environment that the person lived in rather than any specific feelings, thoughts, or behaviors. These strategies were coded into two different categories. Change the environment involved physical changes to the existing environment such as building a ramp onto the house while change to a new environment involved actually relocating to a new environment, such as a first floor apartment or moving in with family. The Kappa coefficient for change to a new environment showed coder agreement of .96 (Intra-class correlation $=.98)$. The strategy of change the environment was not offered in response to the vignettes and therefore neither a Kappa coefficient nor an Intra-class correlation was computed for that specific strategy.

\section{Decisional Outcomes}

Decisional outcomes were coded only for the end-of-life vignette and represent the final decision made in response to the hypothetical situation presented. Cicirelli (1997a) used factor analysis to determine the final two decisional outcomes of end the 
life versus maintain the life. These decisions were given to the participants and they were asked to indicate which decision they would make in that particular situation (Cicirelli, 1997a). Rather than asking participants to indicate acceptance for a decision based on forced choices, the current study addressed the totality of the participant's written response. Five potential decisional outcomes were used: fight against death, accept death, defer the decision to others, end the life through suicide, and end the life through assisted suicide. Each of these decisional outcomes is mutually exclusive and therefore only one outcome was coded for each respondent regardless of the number or type of strategies offered in response to the vignette. Coding the decisions in this way takes into account that not all of the strategies actually suggested a final decision. Some strategies were offered just for the simple reason of managing the situation at hand, rather than thinking ahead to a final solution to the problem (see Appendix $\mathrm{K}$ for the coding manual used for the decisional outcomes). The five decisional outcomes were coded as follows ${ }^{3}$.

The decision of fight against death was coded for all responses that suggested using all resources available and doing all that could be done to avoid the death. The decision of accept death was used when the respondent was clearly offering strategies that suggested resigning themselves to the diagnosis and essentially preparing for the impending death. The decision of defer the decision to others was coded if the respondent suggested turning to others and relying on others for the final decision. The decision of end the life through suicide was coded when the respondent made it clear that there was no other alternative and means should be taken to end the life. The

\footnotetext{
${ }^{3}$ Inter-rater reliability for the decision outcomes resulted in a Kappa of 1.00; therefore, Kappas and Intra-Class Correlations were not computed for the individual outcomes.
} 
decision of end the life through assisted suicide was used when the respondent suggested that other individuals should be involved in ending the life.

The majority of the participant's responses contained a clear outcome such as "don't commit suicide" while others were not as explicitly stated but still contained a clear direction toward one specific outcome. For example, if an individual's response was "get a second opinion and seek all the medical treatment available", it was coded as fight against death. A response of "get your affairs in order, pray, and make things right with God and your family" was coded as accept death; "seek a second opinion from another physician and ask your family for advice" was coded as defer the decision to others; "say his good-byes, make amends with his family, and then make his own choice" was coded as end the life through suicide; and "ask someone very close to you to help end your life" was coded as end the life through assisted suicide.

Although the participant's complete response was used to determine the final decisional outcome, two individuals responding with the same number and type of strategies could ultimately make very different end-of-life decisions. For example, an individual who responded to the end-of-life vignette with "talk with your family, get a second opinion, and if it's really fatal then choose when and how to die" would be coded as having three strategies—seek social support, seek formal support, and change own behavior. After reading the total response of this individual, the final decisional outcome would be coded as end the life through suicide. In contrast, another individual who responds with "find a support group, consult the internet, and live each day to the fullest" would also be coded as having the three strategies of seek social support, seek formal support, and change own behavior. However, in this case this individual's final 
decisional outcome would be coded as accept death. Thus, the problem-solving strategies and decision-making outcomes in the current study, while moderately statistically related—as one would expect—remain conceptually independent. 
Chapter 4: Results

Preliminary Analyses

Preliminary analyses included descriptive statistics (means and standard

deviations) and examination of differences between the two age groups ( $t$-tests and $\left.\chi^{2}\right)$. In addition, correlation coefficients were computed to examine relations among the variables of interest in the current study.

Descriptive Analyses

Descriptive statistics, including means, standard deviations, and frequencies, were computed for the following variables: age, gender, race, marital status, religious affiliation, years of education, difficulty paying bills, religiousness, spirituality, and selfassessed health. The demographics for the total sample, including a contrast between the younger and older adults, are presented in Table 1 and the means and standard deviations for the study variables for the two age groups are presented in Table 2. Independent samples $t$ tests, or chi-square tests when appropriate, were conducted to examine differences between the younger and older adult groups. Independent samples $t$ tests were conducted on the variables coded with an interval level of measurement including: age, years of education, self-assessed health, religiousness, and spirituality. Results of these tests showed significant differences for age, education level, and religiousness in that the older adults in the current sample were more educated and more religious than the younger adults. No significant differences were found for spirituality or self-assessed health (see Tables 1 and 2 for specific results). Chi-square tests were conducted on the variables coded with a categorical level of measurement including: gender, race, marital status, religious affiliation, and difficulty paying bills. 
Results of these tests showed significant differences for race, marital status, religious affiliation, and difficulty paying bills. For example, relative to younger adults, the older adults in the current study were more likely to be Caucasian, married, Protestant, and have no difficulty paying their bills. No significant differences were found for gender (see Tables 1 and 2 for specific results).

Correlational Analyses

Correlation coefficients were computed to address the relation among the independent and dependent variables of interest. Pearson correlation coefficients were computed among the five independent variables of age, gender, organizational religiousness, spirituality, and self-assessed health. As shown in Table 3, age and organizational religiousness were positively related $(r=.37, p<.01)$ In addition, there was a positive association between religiousness and spirituality $(r=.30, p<.01)$. No significant correlations were found between gender or self-assessed health and all other variables.

Correlation coefficients were also computed for the five independent variables and the five mutually-exclusive decisional outcomes. The individual decisional outcomes were coded dichotomously (yes-no), therefore point biserial correlation coefficients were used in this analysis for four of the independent variables. Due to the fact that the independent variable of gender is dichotomous (male-female), and the decisional outcomes are dichotomous, a phi coefficient was computed to examine association of gender with the five decisional outcomes.

As shown in Table 4, age and the decision to fight against death showed a small, negative correlation $\left(r_{p b}=-.21, p<.01\right)$ suggesting that younger adults are more likely 
to choose this end-of-life decision. The decision to accept death shows a small positive correlation with both religiousness and spirituality $\left(r_{\mathrm{pb}}=.28, p<.01\right.$ and $r_{\mathrm{pb}}=.26, p<$ .01 , respectively). Individuals who reported a higher level of religiousness and those who reported a higher level of spirituality were more likely to accept death relative to those who self-reported lower levels of these constructs. In contrast, religiousness shows a small negative correlation with both end the life through suicide and end the life through assisted suicide $\left(r_{\mathrm{pb}}=-.17, p<.05\right.$ and $r_{\mathrm{pb}}=-.24, p<.01$, respectively) while spirituality shows a small negative correlation with end the life through assisted suicide $\left(r_{\mathrm{pb}}=-.18, p<.05\right)$. Although these correlations are small, they suggest that higher levels of religiousness and spirituality are related to a lower endorsement of the decision of suicide-whether personal or assisted. Gender and self-assessed health showed no significant correlations with any of the five end-of-life decisional outcomes.

\section{Hypothesis Testing}

\section{End-of-Life Decisional Outcomes}

Hypothesis 1. Hypothesis 1 stated that no differences were expected between the younger and older adults in the type of end-of-life decision endorsed in response to the end-of-life vignette. The five decisional outcomes used in the current study included: fight against death, accept death, defer the decision to others, end the life through suicide, and end the life through assisted suicide. In the current sample, the most common end-of-life decision endorsed by both the younger and older adults was to accept death (70.2\%). Endorsement for the remaining end-of-life decisions were as follows: $11.8 \%$ chose to end the life through suicide, $5.6 \%$ chose to fight against death, 
$3.4 \%$ chose to end the life through assisted suicide, and $2.2 \%$ chose to defer the decision to others (see Table 5).

To test hypothesis 1 , a chi-square analysis was conducted to determine if there were differences between the younger and older adults on endorsement of the five endof-life decisional outcomes. As shown in Table 5, younger and older adults do endorse different end-of-life decisional outcomes, $\chi^{2}(4, N=166)=11.63, p<.05$. Thus, the null hypothesis was not supported.

To further investigate these differences, a series of post hoc chi-square analyses were conducted addressing age group and each of the five decisional outcomes individually. Of the five decisional outcomes, only the decision to fight against death showed significant age differences, $\chi^{2}(1, N=166)=7.57, p<.01$, with younger adults $(10 \%)$ more likely than older adults $(0 \%)$ to suggest fighting the prognosis and taking advantage of all that medical technology can offer.

One additional post hoc chi-square analysis was conducted to address the within-group differences in endorsement for end-of-life decisions found by Cicirelli (1997a). For this analysis, the older adult group was divided into young-old (55 to 70 years of age) and old-old (71 to 84 years of age). The results of this analysis revealed no within-group differences in older adults when addressing the type of end-of-life decision endorsed, $\chi^{2}(3, N=69)=2.26$, ns. Table 6 presents the number and percent of young-old and old-old adults who endorsed each of the end-of-life decisional outcomes.

Hypothesis 2. Hypothesis 2 stated that acceptance for specific end-of-life decisions would differ as a function of gender, religiousness, spirituality, and self- 
assessed health. Based on the significant results of the chi-square analysis conducted for hypothesis 1 , age was added as an additional predictor to this analysis.

To test hypothesis 2 , the five predictors (i.e., age, gender, religiousness, spirituality, and self-assessed health) and the five decisional outcomes were entered into a multinomial logistic regression. The overall model was significant, with the equation correctly classifying $77 \%$ of the sample, $\chi^{2}(20, N=163)=42.08, p<.01$. Thus, the five independent variables do predict differences in endorsement of decisional outcomes. When examining the roles of the five predictors within the overall model however, only two of the predictors emerged as unique predictors: age and religiousness. Age emerged as a significant predictor of decisional outcome overall, $X^{2}(4, N=163)=9.34, p=.05$, as expected based on the chi square tests conducted for hypothesis 1. Inspection of the Beta coefficients (see Table 7), however, suggest that age did not exert differential prediction across the five decisional outcomes.

Religiousness also emerged as a unique predictor of decisional outcome overall, $\chi^{2}(4, N=163)=11.33, p<.05$. Examination of the standardized regression coefficients (see Table 7) for religiousness revealed that individuals with lower levels of religiousness were significantly more likely to endorse the decision of assisted suicide $(\beta=-.62, p<.05)$. The remaining predictors of gender, spirituality, and self-assessed health did not uniquely predict end-of-life decisional outcomes in the current model (see Table 7).

Hypothesis 3. Hypothesis 3 stated that, although religiousness and spirituality are overlapping constructs, spirituality was expected to have a greater effect on the individual endorsement of end-of-life decisional outcomes over the other four predictors 
of age, gender, religiousness, and self-assessed health. To test this hypothesis, the standardized regression coefficients and odds ratios from the multinomial logistic regression conducted to test hypothesis 2 were examined. Based on the results from the regression analyses, spirituality did not emerge as a unique predictor, $X^{2}(4, N=163)$ $=2.83, \mathrm{~ns}$, of individual end-of-life decisional outcome (see Table 7 ). The standardized regression coefficients for spirituality were small $(\beta<.15)$ in all end-of-life decision types and the Wald statistics were not significant. In addition, the odds ratios for spirituality were small $(O . R . \leq 1.0)$ revealing no significant differential prediction for specific end-oflife decisions. Further inspection of the standardized regression coefficients showed that the regression coefficients for religiousness were of a greater magnitude than spirituality in all decision outcomes. Thus, this hypothesis was not supported.

In addition, the odds ratios were small $(O . R . \leq 1.0)$ for gender revealing no significant differential prediction for specific end-of-life decisions. Although failing to emerge as a unique predictor of end-of-life decision, and as shown in Table 7, the standardized regression coefficients and odds ratios for self-assessed health were of a high magnitude ( $\beta \geq .52$, O.R. $>1.5$; respectively). The failure of these large regression coefficients to emerge as significant may be related to the low percentage of endorsement in some of the decision categories. For example, only 10 individuals out of the total sample chose to fight against death, 21 chose the decision of suicide, and 6 chose assisted suicide. However, even when failing to reach statistical significance, Beta weights and odds ratios can provide important information (Howell, 2003). For example, individuals with better self-assessed health were twice as likely as those 
individuals with poorer self-assessed health to endorse the decision of assisted suicide over the other end-of-life decisional outcomes (O.R. $=2.32)$.

Summary of Decisional Outcomes Hypotheses. In summary, then, the chi square analyses for hypothesis 1 found significant age differences between younger and older adults in the endorsement of specific end-of-life decisions. Post hoc tests revealed that these differences were specific to the decision of fight against death. One additional post hoc analysis revealed no within-group differences in the older adult age group. Therefore, hypothesis 1 was not supported.

The multinomial logistic regression analyses for hypothesis 2 found that the overall model was significant. However, when examining individual contributions of the five independent variables, only age and religiousness emerged as unique contributors of the variance explained for the end-of-life decisional outcomes. No significant effects for gender, spirituality, or self-assessed health emerged in this analysis. Therefore, hypothesis 2 was partially supported, with only religiousness and age emerging as significant predictors of the end-of-life decisional outcomes.

Lastly, the analyses for hypothesis 3 suggest that spirituality does not predict end-of-life decisions to a greater degree than age, gender, religiousness, or selfassessed health. In fact, the standardized regression coefficients for both religiousness and self-assessed health are of a greater magnitude than spirituality. Therefore, hypothesis 3 was not supported.

Religiousness, Spirituality, and Strategies

Hypothesis 4. Hypothesis 4 stated that religiousness and spirituality were expected to be important factors in the high-emotionally salient, end-of-life decisional 
domain but not in the low-emotionally salient consumer domain or the mediumemotionally salient family domain. This hypothesis addressed strategies rather than decisional outcomes; thus, the analyses for hypothesis 4 examined the number and type of strategies generated in response to all three of the problem domains.

The strategies generated in response to the vignettes were categorized according to a coding scheme that included eleven specific strategy types ${ }^{4}$. Using these strategy types, the analyses began with simple frequencies to determine the number and type of strategies the respondents generated across the three decisional domains. In the current sample, the number and type of strategies offered by the participants across all three vignettes were as follows: $74 \%$ sought formal support, $67 \%$ changed their own behavior, 33\% changed another person's cognition, 30\% sought social support, 28\% changed their own cognition, 28\% changed another person's behavior, and $25 \%$ of the sample turned to toward their religion. Fewer than $7 \%$ of the sample generated the remaining strategies of change own emotion, change another person's emotion, and change to a new environment. The final strategy of change the environment was not offered at all by the sample across the three vignettes. In the interest of preserving stability in the measures, only the strategies generated by at least $25 \%$ of the sample were included in the analyses for hypothesis 4 . Thus, the analyses included the seven strategies of religion, change self cognition, change self behavior, change other cognition, change other behavior, seek social support, and seek formal support.

\footnotetext{
${ }^{4}$ The coding scheme for the final analyses only included eleven strategy types. See page 30 for a discussion as to why the strategies of cooperate/discuss and seek information were not included.
} 
To test hypothesis 4, a 2 (Age) $\times 3$ (Domain) $\times 7$ (Strategy) Analysis of Covariance (ANCOVA), with repeated measures on the last two factors, was conducted. This analysis was chosen due to the fact that all of the participants answered the same three vignettes and each vignette was coded according to the same strategy types. The ANCOVA was used to evaluate age differences in strategies generated across three different problem domains and included religiousness and spirituality as covariates. Age (younger, older) was the between-subjects variable in this analysis and problem domain/emotional salience (e.g., consumer-low-emotional salience, family-mediumemotional salience, end-of-life-high-emotional salience) and strategy (e.g., religion, change self cognition, change self behavior, change other cognition, change other behavior, seek social support, seek formal support) were the within-subjects variables. Alpha levels were set at .05 and effects were tested using the multivariate criterion of Wilks's lambda $(\lambda)$.

Examination of the within- and between-subjects effects of the 2(Age) $x$ 3(Domain) $\times 7$ (Strategy) repeated measures ANCOVA revealed a significant three-way interaction of Domain $\times$ Strategy $\times$ Religiousness $^{5}(F(12,1812)=2.31, p<.01)$ showing that the type of strategies generated across the different problem domains vary as a function of the individual's religiousness (see Table 8). To follow up this three-way interaction, separate one-way analyses of variance (ANOVAs) were conducted within each problem domain. Based on the total scores for the religiousness scale, the sample was divided into low, medium, and high levels of religiousness. Those individuals scoring $0-7$ were categorized as having a lower level of religiousness, $8-12$ as

\footnotetext{
${ }^{5}$ Religiousness is a covariate in this analysis, therefore the result is not a true three-way interaction.
} 
medium religiousness, and $13-16$ as higher religiousness. The three levels of religiousness and the seven strategies were then entered into separate one-way ANOVAs and the analyses conducted within each specific domain.

Results from the first ANOVA conducted within the high-emotionally salient, endof-life domain showed significant differences among the three levels of religiousness for the religion strategy $(F(2,164)=6.84, p<.01)$ as well as the change self behavior strategy $(F(2,164)=3.41, p<.05)$. Specifically, post hoc Scheffe tests revealed that those individuals with a higher level of religiousness were more likely to turn toward their religion in response to the end-of-life situation, while those with a lower level of religiousness were more likely to change their own behavior.

Results from the second ANOVA conducted within the medium-emotionally salient, family domain showed significant differences only for the change other behavior strategy $(F(2,164)=3.92, p<.05)$. The post hoc Scheffe tests showed that those individuals with a higher level of religiousness were less likely than those with a medium level of religiousness to try to change another person's behavior. Some non-significant trends also emerged within this domain showing that those individuals with a higher level of religiousness were more likely to try to change their own behavior in response to the family situation while those with a medium level of religiousness were more likely to try to change another person's cognition.

Results from the third ANOVA conducted within the low-emotionally salient, consumer domain show no significant differences among the three levels of religiousness. However, there was a non-significant trend for the seek formal support strategy showing that those individuals with a higher level of religiousness were more 
likely to seek formal support in response to the situation presented than those with a lower level of religiousness.

In addition to the Domain $x$ Strategy $x$ Religiousness interaction, several lowerorder interactions also reached significance (see Table 8). The significant Domain $\mathrm{x}$ Strategy interaction $(F(12,1812)=2.42, p<.01)$ shows that different types of strategies are used across the three different problem domains. Based on a series of paired t-tests of the seven strategies listed in Table 9 (not reported here), results show that the strategy of change self behavior $(M=1.846)$ was the most offered strategy across the three domains followed by seek formal support $(M=1.750)$ and the remaining five strategies. Separate one-way ANOVAs conducted on each strategy across domains reveal that only religion and change self cognition were significantly different $(F(2,152)=$ 5.98, $p<.01, F(2,152)=5.46, p<.01$, respectively). Paired samples t-tests were conducted to determine where those differences lie. Results show that the number of religious strategies in the end-of-life domain was greater than those in both the family and the consumer domains. Results also show that the number of change self cognition strategies was greater in the end-of-life domain, which was greater than those generated for the consumer domain, which in turn was greater than those generated in the family domain.

A Strategy $x$ Age interaction also reached significance $(F(6,906)=10.74, p<$ .001) revealing that younger and older adults generate different strategies across the different problem domains. Post hoc t-tests were conducted to compare the strategies generated by the younger and older adults. As shown in Table 10, significant differences were found for four of the seven strategies. For example, the younger adults 
were more likely to choose the strategies of change self cognition, change self behavior, and change other behavior in response to the situations across domains. In contrast, the older adults were more likely to seek formal support in response to the problem situations.

In addition, the two-way interaction of Domain $x$ Spirituality was also significant $(F(2,302)=3.98, p<.05)$ showing that the strategies generated in response to the vignettes are related to the problem domain and the individual's spirituality. Based on the total scores for the spirituality subscale, the sample was divided into low, medium, and high levels of spirituality. Because the sample mean for spirituality was high ( $M=$ 34.1), and the range of scores for this sample was $15-44$, the divisions were not even. Those individuals scoring $15-32$ were categorized as having a lower level of spirituality, $33-36$ as medium spirituality, and $37-44$ as higher spirituality. Separate one-way ANOVAs were then conducted to address the strategies generated by individuals with differing levels of spirituality within each problem domain. As shown in Table 11, two significant results emerged on the strategies within domains. Significant differences emerged for the religion strategies within the end-of-life domain $(F(2,162)=$ $5.68, p<.01)$. Post hoc Scheffe tests revealed that those individuals with a higher level of spirituality were more likely, than those with a medium or lower level of spirituality, to choose religious strategies in response to the end-of-life situation. Significant differences also emerged for the change self cognition strategies within the consumer domain $(F(2,156)=4.47, p<.05)$. Post hoc Scheffe tests indicate that individuals with a higher level of spirituality were more likely, than those with a lower level of spirituality, to choose the strategy of change self cognition in response to the consumer situation. No 
differences in level of spirituality emerged for the strategies generated in response to the Family situation.

Several main effects and a covariate also emerged as significant in this analysis. A significant main effect for Strategy emerged $(F(6,906)=6.71, p<.001)$ confirming once again that different strategies were generated in response to the different problem situations. The main effect of Domain $(F(2,302)=3.18, p<.05)$ also emerged showing that the domain of the problem, and the emotional salience involved in each situation, influenced the strategies that were generated. Lastly, the covariate of Spirituality reached significance $(F(1,151)=5.27, p<.05)$ revealing that an individual's spirituality influences the choice of strategy used in response to the vignettes, independent of problem domain and age of the individual.

Summary of Repeated-Measures Analysis. In summary, the 2(Age) x 3(Domain) $x 7$ (Strategy) ANCOVA analysis for hypothesis 4 revealed that younger and older adults generate different strategies across the different problem domains and that the strategies generated in response to the vignettes are related to the individual's spirituality. In addition, the strategies also vary as a function of the individual's age and level of religiousness as well as a function of the problem domain. Therefore, the multivariate analysis showed that both religiousness and spirituality are important factors in generating different strategies to solve problems across the varying domains. However, these analyses did not address the pervasiveness of religiousness and spirituality across the three problem domains. Therefore, post hoc analyses focusing on the strategies within the various domains were conducted. 
Hypothesis 4-post hoc analyses. To further analyze the strategies generated in response to the three problem vignettes, post hoc analyses of descriptive frequencies were conducted. This analysis aided in determining the type of strategies that were most frequently offered in the problem-solving process for each vignette. Examination of the frequencies revealed that both the number and type of strategies differed within each the problem domain (See Table 12). For example, in the process of solving the low-emotionally salient consumer decision, $70 \%$ sought formal support, $32 \%$ changed their own behavior, $12 \%$ of the sample changed their own cognition, and $5 \%$ changed another person's cognition. In the medium-emotionally salient family decision, $54 \%$ of the sample changed their own behavior, $31 \%$ changed another person's behavior, $30 \%$ changed another person's cognition, and 19\% sought formal support. Lastly, in the highemotionally salient end-of-life decision, $54 \%$ changed their own behavior, $31 \%$ sought social support, $26 \%$ turned to religion, and $24 \%$ sought formal support.

Once the most frequently-endorsed strategies for each vignette were identified, post hoc analyses were conducted to complete the analyses for hypothesis 4 . A 2 (Age) x 4 (Strategy) repeated measures ANCOVA, with repeated measures on the last factor, was conducted within each problem domain. This analysis evaluated age differences in the type of strategies generated within each problem domain and included religiousness and spirituality as covariates. Age (younger, older) was the between subjects factor and strategy (four most frequently-offered strategies within each domain) was the within subjects factor. Alpha levels for these analyses were set at .05 and the effects were again tested using the multivariate criterion of Wilks's lambda $(\lambda)$. 
The first 2(Age) $\times$ 4(Strategy) ANCOVA was conducted on the high-emotionally salient, end-of-life domain and examined age differences using the four most frequently endorsed strategies (change own behavior, seek social support, religion, and seek formal support) within this domain (See Table 12). Multivariate results for this ANCOVA showed a significant Strategy $\mathrm{x}$ Religiousness interaction, $(F(3,159)=2.86, p<.05 ; \lambda=$ .95) revealing that the endorsement of different types of strategies within this domain was related to the religiousness of the individual. In addition, the Strategy $x$ Age interaction was also significant $(F(3,159)=6.66, p<.001 ; \lambda=.89)$ revealing that older and younger adults generated different numbers of strategies in response to the end-oflife situation. For example, in response to the hypothetical end-of-life situation, the younger adults chose first to change their own behavior then to seek social support, turn toward their religion, and lastly to seek formal support. In contrast, the older adults chose first to change their own behavior then to seek formal support, turn toward their religion, and finally to seek social support. In addition to these results, a main effect of Strategy $(F(3,159)=4.16, p<.01 ; \lambda=.93)$ also emerged.

The within-subjects effects also showed a significant Strategy $\times$ Religiousness interaction $(F(3,483)=3.46, p<.05)$, a Strategy $\times$ Age interaction $(F(3,483)=5.72, p<$ $.01)$ and a main effect of Strategy $(F(3,483)=5.40, p<.01)$. The covariate of Spirituality was also significant $(F(1,161)=4.46, p<.05)$ showing that the choice of strategies generated in response to this vignette was influenced by the individual's spirituality. These results reveal that age is an important factor within this domain and that younger and older adults generate different strategies in response to the end-of-life situation. In addition, the results suggest that both religiousness and spirituality are important factors 
within the high-emotionally salient end-of-life decisional domain. For example, those individuals with higher religiousness and/or higher spirituality were more likely, than those who were less religious or spiritual, to generate a religious strategy in response to the hypothetical end-of-life situation.

The second 2(Age) $x$ 4(Strategy Type) ANCOVA was conducted on the mediumemotionally salient, family domain and examined age differences using the four most common strategies (change own behavior, change another person's behavior, change another person's cognition, and seek formal support) within this domain (See Table 12). The multivariate results of this analysis showed a significant effect for the Strategy $x$ Age interaction $(F(3,159)=4.78, p=.01 ; \lambda=.92)$ revealing that age is an important factor within this domain and younger and older adults generate different strategies in response to the driving domain. For example, the younger adults chose first to change their own behavior then to change another person's behavior, change another person's cognition, and finally to seek formal support. The older adults chose first to change their own behavior then to seek formal support, cooperate/discuss, change another person's cognition and lastly to change another person's behavior. In contrast to the end-of-life decision, religiousness and spirituality did not influence the strategies generated within this domain.

The third 2(Age) $x$ 4(Strategy Type) ANCOVA was conducted on the lowemotionally, salient consumer domain using the four most common strategies (seek formal support, change own behavior, change own cognition, and change other cognition) within this domain (See Table 12). Multivariate results showed a significant Strategy $x$ Age interaction $(F(3,153)=4.53, p<.01 ; \lambda=.92)$ and a significant main 
effect of Strategy $(F(3,153)=7.80, p<.001 ; \lambda=.87)$. The within-subjects results also revealed a Strategy $x$ Age interaction $(F(3,465)=7.02, p<.001)$ as well as a significant main effect of Strategy $(F(3,465)=5.69, p<.01)$. These results reveal, once again, that age is an important factor within this decisional domain and that religiousness and spirituality did not influence the strategies generated.

Summary of post hoc analyses. In summary, then, the post hoc frequency analysis revealed that the most frequently offered types of strategies differed within the various problem domains. The series of 2(Age) x 4(Strategy Type) ANCOVA analyses conducted within each of the domains confirmed that religiousness and spirituality are important in the high-emotionally salient end-of-life decision but not in the lowemotionally salient consumer decision or the medium-emotionally salient family decision. In addition, age emerged as a significant factor across all three domains, revealing that the younger and older adults generated different strategies to solve those specific problems. Therefore, hypothesis 4 is supported in the current study and religiousness and spirituality are not pervasive across multiple decisional domains.

\section{Exploratory Research Question}

The exploratory research question investigated the link between the number and type of strategies generated specifically in response to the end-of-life situation and the decisional outcomes generated from the participant's total written response. To examine whether the different end-of-life decisions were related to a differential use of strategies, a one-way ANOVA (analysis of variance) was conducted using the five mutually exclusive decisional outcomes as the independent variable and the number of strategies generated within the seven strategy types (religion, change own cognition, 
change own behavior, change other cognition, change other behavior, seek social support, and seek formal support) as the dependent variables ${ }^{6}$. Results of the one-way ANOVA show that three of the seven strategy types relate to the endorsement of decisional outcomes, establishing a link between the strategies generated in response to the end-of-life vignette and the participant's specific decision made to solve the problem.

In examining the use of the religion strategies, a significant overall effect emerged $(F(4,150)=3.13, p<.05)$ suggesting that the use of religious strategies does relate to the endorsement of decisional outcomes. However, post hoc Scheffe tests showed that no two decisional outcomes significantly differed from each other within this strategy. In examining the use of the seek social support strategy among the five decisional outcomes, a significant overall effect emerged $(F(4,150)=3.52, p<.01)$ suggesting that the use of this strategy also relates to specific decisional outcomes. The results of the post hoc Scheffe tests revealed a significant difference $(p<.05)$ between those individuals using the strategy of seek social support for the outcome of deferring the decision to others and those who chose the outcome of committing suicide. In examining the use of the strategy to change own behavior, a significant overall effect emerged $(F(4,150)=2.86, p<.05)$ suggesting that the use of this strategy also related to the endorsement of specific decisional outcomes. However, similar to the religion strategies, post hoc Scheffe tests showed that no two decisional outcomes significantly differed from each other. However, there was a non-significant trend $(p<.08)$ between the individuals who chose the outcome of accepting death and those choosing assisted

\footnotetext{
${ }^{6}$ In order to maintain the stability of the analyses in the current investigation, the strategies used in this analysis were limited to the same seven strategies used in the repeated measures ANCOVA for hypothesis four.
} 
suicide. No significant overall effects emerged for the remaining strategies of change own cognition, change other cognition, change other behavior, and seek formal support. Thus, the answer to the exploratory research question - for the current analysis—is that there is a link that can be established between the strategies chosen to handle the situation presented and the final decisions made to resolve the problem. Further investigation should be continued in this area. 


\section{Chapter 5: Discussion}

\section{Overview of the Study}

The current investigation examined the influence of religiousness, spirituality, and emotional salience on decision-making processes and outcomes. In the process of this investigation, three different areas of research were examined: predictors of end-of-life decisions, problem-solving strategies and emotional salience, and the link between problem-solving strategies and decision-making outcomes.

Previous decision-making studies have shown that age and religiousness are important factors in the endorsement of end-of-life decisions (Cicirelli, 1997a). Previous literature has also shown that emotional salience influences problem-solving in that strategies differ as a function of age and problem domain, depending on the emotion involved in the problem presented (Blanchard-Fields et al., 1995). Finally, prior research has shown that linking strategies and outcomes is important to understanding the overall problem-solving process (Patrick \& Strough, 2004). Thus, examining both problem-solving strategies and decision-making outcomes is important because past empirical research has shown consistent age differences in both strategies (BlanchardFields et al., 1995) and end-of-life decisional outcomes (Cicirelli, 1997a).

Based on the importance of this prior research, the current study was designed to replicate and extend each of these areas while concurrently addressing any existing limitations. The current sample included both younger and older adults to more clearly investigate age differences in strategies and end-of-life decisional outcomes. A crosssectional design was chosen to enable a replication of Cicirelli's (1997a) work with older adults' endorsement of end-of-life decisions and Blanchard-Fields et al. (1995) work 
addressing age differences in problem-solving strategies. The format of the decision vignettes replicated Blanchard-Fields et al. (1995) in that each included an open-ended question which enabled the participants to generate their own personal answers to the problems. These personal statements included the number and type of strategies as well as the decisional outcome given in response to the end-of-life vignette. This design improved on Cicirelli's (1997a) study and made it possible to investigate the link between strategies and outcomes. Religiousness and spirituality were included as individual difference variables in relation to both predictors of end-of-life decisions and problem-solving strategies generated in response to the vignettes across the three decisional domains. Thus, using the predictors of age, gender, religiousness, spirituality, and self-assessed health, along with open-ended decisional vignettes varying in emotional salience, allowed the current study to replicate and extend the prior literature in relation to end-of-life decisions, problem-solving strategies, and emotional salience.

\section{Specific Results and Discussion}

\section{Predictors of End-of-Life Decisions}

The first specific aim of this study was to replicate and extend the research of Cicirelli (1997a) on predictors of end-of-life decisions. Cicirelli (1997a) found that endorsement of specific end-of-life decisions differed as a function of the age, gender, and level of religiousness of the participants. However, several design issues limit the usefulness of this research, such as: the exclusion of individuals less than 60 years of age, the use of a lengthy battery of vignettes with close-ended responses, and the use of limited individual difference variables—health, religiousness and additional demographic variables—while neglecting the potential role of spirituality in end-of-life 
decisions (Cicirelli, 1997a). Due to the fact that researchers have continued to examine religiousness and spirituality separately (see Emmons \& Paloutzian, 2003; Hill \& Pargament, 2003; and Zinnbauer, Pargament, \& Scott, 1999), and a study done for the American Association of Retired Persons (AARP; Montenegro, 2004) has shown that religiousness and spirituality are not synonymous; it is important to include both religiousness and spirituality in decision-making studies.

In designing the current investigation, it was determined that further research on additional predictors of end-of-life decisions was needed. Thus, the current study addressed five predictors in end-of-life decision-making (e.g., age, gender, health, religiousness, and spirituality). Findings from a chi-square analysis suggest that younger and older adults endorse different end-of-life decisions. Post hoc chi-square tests suggested that this difference was specific to the decision to fight against death, with the younger adults more likely to endorse this decision than the older adults. However, the difference was small with only $10 \%$ of the younger adults choosing this decision and none of the older adults. These results extend the previous research of Cicirelli (1997a) through the addition of the younger adult group and the finding of differences between the younger and older adults. However, Cicirelli (1997a) did find within-group age differences in the older adults' endorsement for end-of-life decisionsa result that was not replicated in the current analyses. This may have occurred because the current research included a sample of 78 older adults ranging in age from 55 to 84 years of age while Cicirelli (1997a) had a much larger sample including 388 older adults ranging in age from 60 to 100 years of age, allowing a closer look at withingroup differences. In addition, the participants in the current investigation answered 
open-ended questions in their own words rather than choosing from forced choice answers. This format may have allowed the participants to choose a response that may not have been given in Cicirelli's (1997a) original research, thus eliminating the differences among the older adult group.

In addition to age, religiousness was also found to be an important factor in relation to end-of-life decisions. Findings from a multinomial logistic regression suggest that the five predictors of age, gender, religiousness, spirituality, and self-assessed health are associated with an individual's end-of-life decision, with age and religiousness emerging as unique predictors. Individuals who reported a lower level of religiousness were more likely to endorse the decision of assisted suicide, in contrast to those who reported a higher level of religiousness. This result replicates Cicirelli's (1997a) findings which suggested that those individuals who were lower in religiousness were more likely to endorse the decision of ending the life rather than choosing the option of maintaining the life.

Although spirituality was also expected to influence end-of-life decisions, it failed to emerge as a unique predictor in the multinomial logistic regression. This finding suggests that when both religiousness and spirituality are included, religiousness exerts a stronger effect than spirituality with regard to specific end-of-life decisions. Although the Spiritual Transcendence Scale has shown good validity and reliability in previous research (Piedmont, 1999; Piedmont, 2001), it has not been used in research specifically addressing end-of-life decisions. In addition, the Likert-type scale may not have allowed the participants to offer responses reflecting their true level of spirituality. Another explanation may be that the social support gained from membership or 
involvement in a religious organization outweighs one's subjective connection to the transcendent when making these important decisions; therefore spirituality does not matter. Although this explanation cannot be addressed here, including spirituality as a predictor of end-of-life decisions does extend the work of Cicirelli (1997a) and these unanswered questions stress the importance of continuing this line of research in the future.

Additional predictors addressed in the multinomial logistic regression included gender and self-assessed health. Cicirelli (1997a) found that men favored the decision of ending the life while women favored the decision of maintaining the life or deferring the decision to others. This result was not replicated in the current study, possibly due to the open-ended format of the vignettes. Using an open-ended format rather than forced-choice answers may have allowed the men and women in this study to generate responses that were more similar than different. It may be that when choosing an endof-life decision that is not a forced-choice answer, men and women think alike and gender just does not matter. It may also be that Cicirelli's (1997a) gender effect was really a proxy for some other measure such as religiousness or spirituality. Prior research has shown that women tend to be more involved in religious activities (Peacock \& Poloma, 1999) and that women are more spiritual than men across the life span (Wink \& Dillon, 2002). Therefore, although gender did not emerge as significant in this study, it should not be eliminated from further research. Instead, the results suggest that the gender differences found in Cicirelli's (1997a) research with older adults should be investigated further. Due to the age range of the older adults in Cicirelli's (1997a) research, it is possible that the results found in his research were due to individual 
differences in beliefs across the generations rather than just age differences. Different cohorts may have very different ideas about what is acceptable, or not acceptable, in regard to end-of-life decisions.

Cicirelli (1997a) reported that those individuals with poor self-assessed health were more likely to endorse the decision of ending the life. Although failing to reach statistical significance in the current analysis, the standardized regression coefficients and the odds ratios in the multinomial logistic regression imply that self-assessed health may influence end-of-life decisions. For example, the odds ratios for self-assessed health were greater than 1.5 in all of the decision outcomes. This result suggests that those individuals with better self-assessed health were one and a half times more likely to endorse all of the end-of-life decisions used in this analysis-more so than those with poorer self-assessed health. The fact that so few individuals endorsed the decisions of fight against death $(n=10)$, suicide $(n=21)$, and assisted suicide $(n=6)$ may explain why the regression coefficients were not significant. However, other variables could also be involved-a value such as experience--that influence the individuals with better selfassessed health to lean toward the decision of ending the life. Although these issues cannot be answered in the current investigation, further research in this area is warranted to address the issue of self-assessed health in relation to end-of-life decisions.

Problem-Solving Strategies and Emotional Salience

The second specific aim of this study was to replicate and extend the research of Blanchard-Fields et al. (1995) on problem-solving strategies and emotional salience. Using a broad age range, Blanchard-Fields et al. (1995) found that the strategies 
generated in response to problem-solving vignettes differed as a function of both the age of the individual and the domain of the problem. However, Blanchard-Fields et al. (1995) also had some limitations in that additional individual difference variables such as religiousness and spirituality were not assessed. Given the importance of religion and spirituality in the lives of many adults, the addition of religiousness and spirituality extends the problem-solving literature by including individual difference variables that have not been examined in relation to problem-solving strategies. Such efforts may increase the ecological validity of the tasks as well as increase the generalizability of research findings. Thus, further research in this area was warranted to extend the existing literature and examine the influence of additional individual difference variables in relation to problem-solving strategies and emotional salience.

Age differences were examined in the current investigation in both the number and type of strategies generated in response to three decisional domains with differing levels of emotional salience. Findings from a 2(Age) $\times 3$ (Domain) $\times 7$ (Strategy) repeated measures analysis of covariance (ANCOVA) omnibus test suggest that age is an important factor in problem-solving. The younger and older adults in the current study generated different numbers of strategies across the different problem domains. In addition, further examination of the analysis of covariance revealed that the type of strategies generated across the three vignettes also varied as a function of age. For example, the younger adults chose to change their own behavior, seek formal support, change another person's cognition, and change their own cognition while the older adults chose to seek formal support, change their own behavior, turn toward religion, and seek social support. These findings are a replication of Blanchard-Fields et al. 
(1995) in that age differences were found in problem-solving style across the various vignettes with the younger adults preferring to use more problem-focused strategies and the older adults preferring to use more emotion-focused strategies. These results are important in understanding that younger and older adults may choose very different strategies in solving similar problems. Neither is right or wrong, but each is unique to the age of the individual.

Based on the results from Blanchard-Fields et al. (1995), age and emotional salience interact on both the number and type of strategies generated in response to the problem-solving vignettes. These results were replicated in the current study and age differences in the strategies generated within each of the specific problem domains were also found. Post hoc analyses of covariance conducted within each problem domain suggest that age is important in the choice of strategies generated in response to the specific problems presented. In response to the high-emotionally salient end-oflife domain, both the younger and older adults chose to turn toward their religion, change their own behavior, seek social support, and seek formal support. However, there were differences in both the number and type of strategies generated. For example, the younger adults chose first to change their own behavior then to seek social support, turn toward their religion, and lastly to seek formal support. In contrast, the older adults chose first to change their own behavior then to seek formal support, turn toward their religion, and finally to seek social support.

In the medium-emotionally salient family domain, both the younger and older adults chose to change their own behavior, change another person's cognition, change another person's behavior, and seek formal support. However, these strategies again 
differed in number and type with the younger adults choosing first to change their own behavior, then to change another person's behavior, change another person's cognition, and finally to seek formal support. The older adults chose to change their own behavior first and then to seek formal support, change another person's cognition, and finally to change another person's behavior. In the low-emotionally salient consumer decision, both the younger adults and the older adults chose to seek formal support, change their own behavior, change their own cognition, and change another person's cognition. No differences emerged in either number or type of strategy generated in this domain.

Thus, findings from the analysis of covariance suggest that age matters when choosing strategies—specifically in response to the high- and medium-emotionally salient domains_-and that younger and older adults make different choices when solving similar problems. However, the results of the current study did not find these differences for the low-emotionally salient domain. These results are different from Blanchard-Fields et al. (1995) in that age differences were found in the low- and medium-emotionally salient vignettes, but not in the high-emotionally salient vignettes. The differences in the high-emotionally salient vignette may be due to the end-of-life scenario which brings up many different issues that may not be included in similar highly emotional decisions such as relocation or caregiving. Therefore, the current results are a partial replication of Blanchard-Fields et al. (1995) and an extension of that work by suggesting that younger and older adults differed in their responses to the highemotionally salient end-of-life vignette. These results are important in understanding that younger and older adults may choose very different strategies depending on their age and the level of emotion involved in the situation. Again, neither is right or wrong, 
but the knowledge that individuals choose different strategies in different situations may make our understanding of problem-solving more complete.

While the current findings suggest that the age of the individual and the domain of the problem are important when generating strategies to specific problem situations, there are other factors that need to be considered. The current study extended the research of Blanchard-Fields et al. (1995) by including two individual difference variables not previously addressed—-those of religiousness and spirituality. Findings from the analysis of covariance suggest that the strategies generated in response to the decision vignettes were related to the problem domain but also to the individual's spirituality. However, spirituality is not the only individual difference variable that matters when generating strategies. Religiousness also is important. For example, findings suggest that the type of strategies generated across the problem domains also differ depending on the individual's religiousness, extending Cicirelli's (1997a) results with end-of-life decisions. Thus, individuals do take their religiousness and spirituality into consideration when deciding on which strategies to use in problem-solving situations. Knowledge of how religiousness and spirituality are involved in choosing strategies to solve everyday problems adds to a more complete understanding of the problemsolving process. Thus, additional research in this area is warranted to further examine the influence of religiousness and spirituality in problem-solving strategies.

\section{Decision-Making Processes and Outcomes}

The third specific aim of this study was to build on the logic of Patrick and Strough (2004) and address an exploratory question as to whether a link could be established between problem-solving strategies and decision-making outcomes. Patrick 
and Strough (2004) demonstrated that strategies generated in response to hypothetical vignettes could be linked to intended real-world behavior. Their results show that the individual difference variable of experience is related to the participant's intentions to relocate in that those older adults who had experience with this problem were able to generate more strategies in response to the relocation vignettes. Although the decision to relocate may include a high level of emotion for many individuals, Patrick and Strough (2004) did not include younger adults, nor did they examine multiple domains. Additional research is warranted to investigate whether additional individual difference variables—such as religiousness and spirituality—influence a range of highly emotional decisions and whether a link can be established between strategies and outcomes.

Findings from one-way analyses of variance (ANOVAs) in the current study suggest that the three strategies of religion, change own behavior, and seek social support generated in response to the end-of-life vignette do relate to specific decisional outcomes. In terms of the strategies generated in response to the end-of-life vignette, the strategy of religion was suggested by fewer than one-third of the sample. Those individuals using this strategy were more likely to choose the outcomes of accepting death or deferring the decision to someone else. Change own behavior was the most used strategy overall, but was still not utilized very often ( $53 \%$ of the total sample). The majority of individuals who chose to use this strategy were more likely to endorse the decision of accepting the diagnosis of death or changing their behavior to commit assisted suicide. Surprisingly, the strategy to seek formal support (i.e. hospice care or physician's advice) was rarely offered, as was the strategy to seek social support (i.e., talk with family or friends). Similar to those who suggested religious strategies, 
individuals suggesting social support were more likely to defer their end-of-life decision to someone else.

To address the link between strategies and outcomes further, post hoc Scheffe tests were conducted. These tests indicate that only the strategy of seek social support showed significant differences across the decisional outcomes while the strategy of change own behavior showed only a non-significant trend. For example, those individuals who would defer the end-of-life decision to others were most likely to endorse the strategy of seeking social support. In contrast, those individuals choosing to accept death were most likely to endorse the strategy of changing their own behavior. These results are important because little previous research has addressed the relation among specific strategies and the final outcome of the problem-solving or decisionmaking process. Problem-solvers are likely to choose strategies that help them reach their final decision, thus, when prior research has catalogued age differences in strategies, it has neglected to consider whether younger and older adults held the same outcome in mind. The current study suggests that this question needs additional research in order to determine whether younger and older adults may indeed choose different final decisions.

The finding that three of the seven strategies generated in response to the endof-life situation significantly link to the decisional outcomes extends the work of Patrick and Strough (2004). Patrick and Strough (2004) were able to link strategies to intended real-world behavior while the current study shows a link between strategies generated in the lab and the final outcomes generated by the participant's total response to the problem presented. The current findings stress the importance of addressing this link in 
other areas of research and in other rare, highly emotional problems. The results also extend the work of Cicirelli (1997a) who only addressed end-of-life decisional outcomes and the work of Blanchard-Fields et al. (1995) who only addressed strategiesemphasizing once again that in order to understand the overall problem-solving and decision-making process, researchers need to address both strategies and outcomes and search for a link between the two.

\section{Advancing the Field}

\section{Religiousness and Spirituality}

Religiousness and spirituality have been shown to be separate, but related constructs in that religiousness represents a narrow construct encompassing the behaviors and practices involved in religion such as church attendance, while spirituality represents a more subjective experience without the outward expression of religious behaviors (Hill \& Pargament, 2003). The current study investigated the potential role of religiousness and spirituality in both end-of-life decisions and strategies used in everyday problem-solving. While religiousness has been addressed in prior literature of end-of-life decisions (Cicirelli, 1997a), spirituality has not. In addition, this is the first study to address these constructs in the area of everyday problem-solving. Findings suggest that both religiousness and spirituality are important in some decisional domains. For example, religiousness is important when making rare, highly emotional end-of-life decisions and emerged as a unique predictor in the multinomial logistic regression. These findings replicate Cicirelli's (1997a) results and emphasize the importance of considering an individual's religiousness when addressing predictors of end-of-life decisions. Spirituality did not emerge as a unique predictor in this analysis, 
although the use of this predictor still extends the work of Cicirelli (1997a) suggesting that this variable should be examined further in relation to end-of-life decisions.

In contrast to the emphasis of religiousness in predicting end-of-life decisions, both religiousness and spirituality may be important in the selection of strategies used to deal with the situation at hand. For example, spirituality emerged as a significant covariate and religiousness emerged in a significant Domain $\times$ Strategy $\times$ Religiousness three-way interaction in the repeated measures ANCOVA. These findings extend the work of Blanchard-Fields et al. (1995) and advance the field by showing that although religiousness and spirituality are overlapping constructs, they may have differential effects in relation to strategies and outcomes. Due to the importance of religion and spirituality in many individual's lives, continued research with both religiousness and spirituality is warranted. Thus, future research addressing both problem-solving strategies and decision-making outcomes needs to emphasize the importance of including the individual difference variables of religiousness and spirituality when examining the decision-making process.

Current findings show that the effects of religiousness and spirituality are not pervasive across decisional domains and therefore suggest that an individual is more likely to turn to their religious or spiritual beliefs when trying to solve highly emotional problems. This makes sense in that most individuals, even if they are very religious or very spiritual, are not likely to talk to their pastor or pray for help when returning merchandise to a store or when trying to convince a family member that they should no longer drive. Results from the post hoc analyses of covariance within each of the decisional domains revealed that religious strategies were generated in response to the 
end-of-life domain, but not in response to the consumer or the family domain.

Religiousness and spirituality are very prominent to some individuals; and those with higher levels of religiousness and/or spirituality will consider these issues before making a rare and emotional decision. Feelings of control may also factor in as most individuals facing the end of their life may feel a lack of control and therefore turn to God, or a higher power, for help (Cicirelli, 1997a), increasing the influence of religiousness and spirituality in these highly emotional decisions.

Everyday Problem-Solving and End-of-Life Decisions

The results from this study advance the field of problem-solving and decisionmaking in several important ways. For example, the current study extends Cicirelli's (1997a) research by including the construct of spirituality as a predictor of end-of-life decision. Results suggest that in order to predict an individual's end-of-life decision, the age of the individual and their religious and/or spiritual beliefs need to be taken into consideration. In addition, religiousness and spirituality were added as constructs to the area of everyday problem-solving, extending the work of Blanchard-Fields et al. (1995). Findings suggest that although both constructs are important in choosing strategies to solve everyday problems, they are not pervasive across decisional domains. Finally, current findings show that three of the problem-solving strategies generated in response to the high-emotionally salient end-of-life vignette do relate to the final decisional outcomes. This result extends Patrick and Strough's (2004) findings suggesting that future studies in the area of problem-solving and decision-making should include an examination of both strategies and outcomes and continue to research the link between the two. These results emphasize the importance of including other individual difference 
variables such as religiousness and spirituality in relation to different decisions across problem domains. Although these results advance the field, additional unanswered questions remain and further research is needed to examine how these predictors do, and do not, vary across domains and/or emotional salience.

\section{Limitations}

Several caveats should be noted when interpreting the results from the current study, including aspects of the sample and the measures. The individual characteristics addressed in the sample were limited to age, gender, religiousness, spirituality, and self-assessed health. Future studies examining predictors of end-of-life decisions and the use of problem-solving strategies in response to decision vignettes should consider the addition of other constructs such as personality, depression, happiness, and chronic health conditions. For example, despite the sample's relatively good health, the odds ratio showed that those adults higher in self-assessed health were more likely to choose assisted suicide. These results show the need for further investigation, specifically in the area of self-assessed health.

In addition to these characteristics, other age groups should also be included in future investigations. Results from this study are limited to younger and older adults. Including additional age groups such as adolescents or middle-aged adults would contribute to the study's generalizability. One other potential limitation to the sample is history effects. Due to the combined sample of data that was collected in 2002 and in 2004, the older adults from 2004 may have been exposed to media or other end-of-life influences that the older adults from 2002 were not privy to. An independent samples $t$ test was conducted between the two samples and showed no differences in the 
variables of interest to this study. However, there may have been additional differences in individual variables that were not included in this study.

Potential limitations in the measures also need to be noted. The demographic variables used in this study were chosen because they had shown effects in the previous literature addressing both problem-solving strategies (Blanchard-Fields et al., 1995) and decisional outcomes (Cicirelli, 1997a). The measures selected for the study were appropriate to address the hypotheses in question and aided in teasing apart the constructs of religiousness and spirituality. The measures used for each of these constructs had been tested empirically in prior research demonstrated good internal consistency. The Spiritual Transcendence Scale (Piedmont, 1999) as a whole includes three subscales. The current investigation used only one of these subscales to measure spirituality and therefore the results may have shown weakened effects of spirituality. Although this limitation cannot be addressed in the current investigation, future research should consider the use of the Spiritual Transcendence Scale in its entirety.

Another potential limitation is the use of a self-administered survey format. Surveys do have advantages in that they can bring items to light that are not directly observable in the participants (Dillman, 2000). However, there is also less experimental control because the participants are completing the surveys without assistance from researchers. In addition, a survey format may include the limitation of socially desirable responses (Dillman, 2000). Due to the sensitive nature of the vignettes, however, the survey format may have actually added to the participant's assurance of confidentiality and reduced the biases in their responses. For example, a participant may have been more open about their final end-of-life decision because they were able to write it down 
in a confidential survey rather than conversing with an interviewer. Future studies may choose the alternative of an interview format which does increase the experimental control of the study. However, there are limitations to this format also as leading questions from the researcher may add to both the experimenter and responder biases. Other potential limitations include the hypothetical problems examined in this study and the use of only three domains. The problem vignettes used in the current study were ambiguous, very much like the ill-structured problems that are seen in daily life. These problems allowed the participants to generate their own responses as to how the individual in the vignette should solve the problem. Cicirelli (1997a) used seventeen vignettes; however each was very detailed and asked the participant to choose an endof-life option based on how they themselves would solve the problem. This was a limitation in his study in that the participants may have experienced confusion with the detail of the vignettes or fatigue with the number of problems. In addition, the participants were each answering the questions based on how they would individually solve the problem. The problem vignettes used in the current study asked the participants to respond to the question based on how the actor in the vignette should solve the problem. One other potential limitation is that each hypothetical vignette represented one specific problem-solving domain and one level of emotional salience. Future studies should include several different domains in each level of emotional salience to examine whether the results generalize to different types of problems within the same domain and the same level of emotional salience. For example, it is not known if the effects of religiousness and spirituality found in response to the end-of-life 
domain would be replicated in other high-emotionally salient problems, such as relocation or caregiving, or if the effect is restricted only to an end-of-life decision.

Potential limitations with the coding scheme for the strategies and outcomes should also be noted. The scheme used to code the strategies generated in response to the vignettes has been well established in the prior literature (Berg et al., 1998; Strough, Patrick, \& Swenson, 2003; Swenson, 2003; Patrick \& Strough, 2004). Although one might suggest coding limitations with the strategies indicated in the coding scheme, this situation did not become an issue. In addition, the inter-rater reliability established between the coders added to the stability of both the coding scheme and the results of the investigation. In contrast to the thirteen strategies used to code the responses to all three vignettes, the decisional outcomes were coded according to a range of five possible end-of-life decisions. This range builds on the factor analysis used in Cicirelli's (1997a) research and allowed the current study to address additional end-of-life options rather than simply maintaining or ending the life. As with the strategies, one could suggest that other end-of-life options should have been considered. However, an interrater reliability of $100 \%(K=1.00)$ was achieved on the decisional outcomes coded in response to the hypothetical end-of-life vignette, therefore the use of additional outcomes was not an issue.

Lastly, limitations to power need to be discussed. The sample size was large enough to provide sufficient power to detect medium effect sizes in the analyses conducted, the alpha level was set at .05, and the multivariate criterion of Wilks's lambda $(\lambda)$ was used. However, the number of analyses conducted in this study may have increased Type I error thereby increasing the danger of accepting a result as 
significant when it is not. While conducting multiple analyses does increase Type I error, many of the analyses in the current study returned non-significant results. Given that only one significant result was expected by chance alone, and more than one result reached significance, the increase in Type I error was not considered to be a problem in this study.

\section{Conclusions and Future Directions}

Despite the limitations to the sample and the measures, this study has answered some important questions about the decision-making process. First, the findings show that there are differences in how younger and older adults approach problem-solving and decision-making. The problem-solving strategies and decisional outcomes of both younger and older adults differ depending on the age of the individual. However, it is not known if these differences continue across the lifespan. Thus, these differences need to be addressed further, perhaps in a longitudinal format in which the changes in decisionmaking can be addressed over time. Second, religiousness and spirituality were shown to have an impact on the decision-making process, influencing both the strategies used to solve the problem and the decisions made in response to the end-of-life decision. According to the results of this study, the effects of religiousness and spirituality are not pervasive across the three domains examined. Future studies need to address other high-emotionally salient decisions to determine if the effects of religiousness and spirituality impact other emotional decisions as well or if an end-of-life domain is a special case. In addition, other low- and medium-emotionally salient problems should be included to determine whether the effects of religiousness and spirituality are pervasive to other problems within these domains. Third, this study suggests that future research 
needs to continue to establish a link between problem-solving strategies and decisional outcomes. Current findings show that some of the strategies generated to solve the end-of-life domain relate to the end-of-life decision made in response to the vignette. Continuing to research this link will answer more questions and lead to a more complete understanding of the decision-making process specifically for individuals facing the end of their life.

Although this study answered some important questions, many more remain. For example, the relation of additional individual characteristics to the decision-making process should be considered in future problem-solving and decision-making studies. In addition, questions addressing other constructs such as quality of life, future time perspective, and a better measure of self-assessed health and spirituality should also be considered. Continuing to investigate individual difference variables will advance the field and enable a more complete understanding of the individual decision-making process. Finally, future studies should also include additional vignettes varying in levels of emotional salience across different problem domains to determine whether the effects of religiousness and spirituality are pervasive across all domains or only influence high-emotionally salient problems.

In conclusion, then, the results of the current study have advanced the field in three important ways. First, this study replicated and extended the work of Cicirelli (1997a) by showing that age, religiousness, and spirituality are important predictors in end-of-life decisions. Second, this study partially replicated and extended the work of Blanchard-Fields and Colleagues (1995) by being the first to suggest that religiousness and spirituality are important individual difference variables in the problem-solving 
process. These results are important particularly when solving high-emotionally salient problems such as end-of-life, but are not pervasive across other decisional domains. Finally, this study extended the work of Patrick and Strough (2004) by demonstrating that a link can be established between problem-solving strategies and decision-making outcomes. 


\section{References}

Allaire, J. C., \& Marsiske, M. (2002). Well- and ill-defined measures of everyday cognition: Relationship to older adults' intellectual ability and functional status. Psychology and Aging, 17, 101-115.

Berg, C. A., Meegan, S. P., \& Klaczynski, P. (1999). Age and experiential differences in strategy generation and information requests for solving everyday problems. International Journal of Behavioral Development, 23, 615-639.

Berg, C. A., Strough, J., Calderone, K. S., Sansone, S., \& Weir, C. (1998). The role of problem definitions in understanding age and context effects on strategies for solving everyday problems. Psychology and Aging, 13, 29-44.

Blanchard-Fields, F. (1986). Reasoning on social dilemmas varying in emotional saliency: An adult developmental perspective. Psychology and Aging, 1, 325333.

Blanchard-Fields, F. (1998). The role of emotion in social cognition across the adult life span. In K. W. Schaie \& M. P. Lawton (Eds.), Annual review of gerontology and geriatrics: Focus on emotion and adult development (pp. 238-265). New York: Springer Publishing.

Blanchard-Fields, F., Chen, Y., \& Norris, L. (1997). Everyday problem solving across the adult life span: Influence of domain specificity and cognitive appraisal. Psychology and Aging, 12, 684-693.

Blanchard-Fields, F., Chen, Y., Schocke, M., \& Hertzog, C. (1998). Evidence for context-specificity of causal attributions across the adult life span. Aging, Neuropsychology, and Cognition, 5, 241-263. 
Blanchard-Fields, F., Jahnke, H. C., \& Camp, C. (1995). Age differences in problemsolving style: The role of emotional salience. Psychology and Aging, 10, 173-180.

Bonner, K., Koven, L. P., \& Patrick, J. H. (2003). Effects of religiosity and spirituality on depressive symptoms and prosocial behaviors. Journal of Religious Gerontology, $14,189-205$.

Cicirelli, V. G. (1997)a. Relationship of psychosocial and background variables to older adults' end-of-life decisions. Psychology and Aging, 12, 72-83.

Cicirelli, V. G. (1997)b. Elders' end-of-life decisions: Implications for hospice care. The Hospice Journal, 12, 57-73.

Cicirelli, V. G. (1998). Views of elderly people concerning end-of-life decisions. The Journal of Applied Gerontology, 17, 186-203.

Cicirelli, V. G., MacLean, A. P., \& Cox, L. S. (2000). Hastening death: A comparison of two end-of-life decisions. Death Studies, 24, 401-419.

Cornelius, S. W., \& Caspi, A. (1987). Everyday problem solving in adulthood and old age. Psychology and Aging, 2, 144-153.

Denney, N. W., \& Pearce, K. A. (1989). A developmental study of practical problem solving in adults. Psychology and Aging, 4, 438-442.

Diehl, M., Willis, S. L., \& Schaie, K. W. (1995). Everyday problem solving in older adults: Observational assessment and cognitive correlates. Psychology and Aging, 10, 478-491.

Dillman, D. A. (2000). Mail and internet surveys: The tailored design method. New York, NY: Wiley and Sons, Inc. 
Emmons, R. A., \& Paloutzian, R. F. (2003). The psychology of religion. Annual Review of Psychology, 54, 377-402.

The Gallup Organization (2005). Retrieved January 23, 2006, from http:// brain.gallup.com/content/default.aspx

Harrison, M. O., Koenig, H. G., Hays, J. C., Eme-Akwari, A. G., \& Pargament, K. I. (2001). The epidemiology of religious coping: A review of recent literature. International Review of Psychiatry, 13, 86-93.

Hill, P. C., \& Pargament, K. I. (2003). Advances in the conceptualization and measurement of religion and spirituality: Implications for physical and mental health research. American Psychologist, 58, 64-74.

Howell, D. C. (2002). Statistical methods for psychology ( $5^{\text {th }}$ ed.). California: Duxbury.

Kenney, B. P., Vaughn, C. E., \& Cromwell, R. E. (1977). Identifying the socio-contextual forms of religiosity among urban ethnic minority group members. Journal for the Scientific Study of Religion, 16, 237 - 244.

Krause, N., Chatters, L. M., Meltzer, T., \& Morgan, D. L. (2000). Using focus groups to explore the nature of prayer in late life. Journal of Aging Studies, 14, $191-212$.

Lawton, M. P., Moss, M., Fulcomer, M., \& Kleban, M. H. (1982). A research and serviceoriented Multilevel Assessment Instrument. Journal of Gerontology, 37, 91-99.

Marsiske, M., \& Willis, S. L. (1995). Dimensionality of everyday problem solving in older adults. Psychology and Aging, 10, 269-283.

McCullough, M. E., Hoyt, W. T., Larson, D. B., Koenig, H. G. Thoresen, C. (2000). Religious involvement and mortality: A meta-analytic review. Health Psychology, 19, 211-222 
Meyer, B. J. F., Russo, C., \& Talbot, A. (1995). Discourse comprehension and problem solving: Decisions about the treatment of breast cancer by women across the life span. Psychology and Aging, 10, 84-103.

Mills, T. L. \& Wilmoth, J. M. (2002). Intergenerational differences and similarities in lifesustaining treatment attitudes and decision factors. Family Relations, 51, 46-54.

Montenegro, X. P. (2004). Spirituality and religion among Americans age 45 and older. Conducted for AARP The Magazine. National Member Research Knowledge Management.

Pargament, K. I. (1992). Of means and ends: Religion and the search for significance. The International Journal for the Psychology of Religion, 2, 201-229.

Pargament, K. I. (2000). The psychology of religion and spirituality? Yes and no. The International Journal for the Psychology of Religion, 9, 3-16.

Pargament, K. I., Kennell, J., Hathaway, W., Grevengoed, N., Newman, J., \& Jones, W. (1988). Religion and the problem-solving process: Three styles of coping. Journal for the Scientific Study of Religion, 27, 90-104.

Patrick, J. H. (2000). Decision making in late adulthood (NIA R03 Ag19917). Unpublished manuscript. Department of Psychology, West Virginia University.

Patrick, J. H., \& Strough, J. (2004). Everyday problem solving: Experience, strategies, and behavioral intentions. Journal of Adult Development, 11, 9-18.

Peacock, J. R., \& Poloma, M. M. (1999). Religiosity and life satisfaction across the life course. Social Indicators Research, 48, 321-345. 
Piedmont, R. L. (1999). Does spirituality represent the sixth factor of personality? Spiritual transcendence and the five-factor model. Journal of Personality, 67, 985-1014.

Piedmont, R. L. (2001). Spiritual transcendence and the scientific study of spirituality. Journal of Rehabilitation, 67, 4-14.

Reese, H. W. (1992). Problem solving by algorithms and heuristics. In S. C. Hays \& L. J. Hays (Eds.), Understanding verbal relations (pp. 153-179). Reno, NV: Context Press.

Sinnott, J. D. (1998). The development of logic in adulthood: Postformal thought and its applications. New York: Plenum.

Strough, J., Berg, C. A., \& Sansone, C. (1996). Goals for solving everyday problems across the life span: Age and gender differences in the salience of interpersonal concerns. Developmental Psychology, 32, 1106-1115.

Strough, J., Patrick, J. H., \& Swenson, L. (2003). Strategies for solving everyday problems faced by grandparents: The role of experience. In B. Hayslip \& J. H. Patrick (Eds.), Working with custodial grandparents. New York, NY: Springer Publishing.

Swenson, L. (2003). Adolescent friendship, relationship quality, and delinquency: Associations with social and cognitive problem-solving performance. Dissertation Abstracts International: Section B: The Sciences and Engineering, 65, p. 466.

Watson, T. L., \& Blanchard-Fields, F. (1998). Thinking with your head and your heart: Age differences in everyday problem-solving strategy preferences. Aging, Neuropsychology, and Cognition, 5, 225-240. 
Wellman, R. J., \& Sugarman, D. B. (1999). Elder and young adults' perceptions of the decision to withdraw from medical treatment: A replication and extension. Journal of Social Behavior \& Personality, 14, 287-298.

Willis, S. L., Dolan, M. M., \& Bertrand, R. M. (1999). Problem solving on health-related tasks of daily living. In D. C. Park, R. W. Morrell, \& K. Shifren (Eds.), Processing of medical information in aging patients: Cognitive and human factors perspectives (pp. 199-219). Hillsdale, NJ: Erlbaum.

Wink, P., \& Dillon, M. (2002). Spiritual development across the adult life course: Findings from a longitudinal study. Journal of Adult Development, 9, 79-94.

Wink, P., \& Dillon, M. (2003). Religiousness, spirituality, and psychosocial functioning in late adulthood: Findings from a longitudinal study. Psychology and Aging, 18, 916-924.

Yates, J. F., \& Patalano, A. L. (1999). Decision making and aging. In D. C. Park, R. W. Morrell, \& K. Shifren (Eds.), Processing of medical information in aging patients: Cognitive and human factors perspectives (pp. 31-54). Hillsdale, NJ: Erlbaum.

Zinnbauer, B. J., Pargament, K. I., Cole, B., Rye, M. S., Butter, E. M., Belavich, T. G., Hipp, K. M., Scott, A. B., \& Kadar, J. L. (1997). Religion and spirituality: Unfuzzying the fuzzy. Journal for the Scientific Study of Religion, 36, 549-564.

Zinnbauer, B. J., Pargament, K. I., \& Scott, A. B. (1999). The emerging meanings of religiousness and spirituality: Problems and prospects. Journal of Personality, 67, 889-919. 
Table 1

Demographic Differences by Age Group

\begin{tabular}{|c|c|c|c|c|c|}
\hline & $\underline{\text { Total Sample }}$ & Younger Adults & Older Adults & & \\
\hline Characteristic & $\%(N=178)$ & $\%(n=100)$ & $\%(n=78)$ & $\underline{\mathrm{df}}$ & $\mathrm{t}$ or $\chi^{2}$ \\
\hline Gender & & & & 1 & 1.50 \\
\hline Men & 41.0 & 45.0 & 35.9 & & \\
\hline Women & 59.0 & 55.0 & 64.1 & & \\
\hline Race & & & & 2 & $11.85^{\star *}$ \\
\hline Caucasian & 92.1 & 86.0 & 100.0 & & \\
\hline African American & 3.9 & 7.0 & 0.0 & & \\
\hline Other & 3.9 & 7.0 & 0.0 & & \\
\hline Marital Status & & & & 3 & $154.90^{* *}$ \\
\hline Married & 27.0 & 1.0 & 60.3 & & \\
\hline Widowed & 9.6 & 0.0 & 21.8 & & \\
\hline Divorced & 4.5 & 0.0 & 10.3 & & \\
\hline Single, & 56.7 & 97.0 & 5.1 & & \\
\hline \multicolumn{6}{|l|}{ Never Married } \\
\hline Did Not Respond & 2.2 & 2.0 & 2.5 & & \\
\hline Religious Affiliation & & & & 12 & $36.94^{* *}$ \\
\hline Protestant & 48.9 & 35.0 & 66.7 & & \\
\hline Catholic & 21.3 & 28.0 & 12.8 & & \\
\hline Baptist & 3.4 & 6.0 & 0.0 & & \\
\hline
\end{tabular}




\section{(Table 1 continued)}

\begin{tabular}{|c|c|c|c|c|c|}
\hline & Total Sample & $\underline{\text { Younger Adults }}$ & $\underline{\text { Older Adults }}$ & & \\
\hline Characteristic & $\%(N=178)$ & $\%(n=100)$ & $\%(n=78)$ & $\underline{\mathrm{df}}$ & $\underline{\operatorname{tor} \chi^{2}}$ \\
\hline Methodist & 1.7 & 3.0 & 0.0 & & \\
\hline Jewish & 3.9 & 3.0 & 5.1 & & \\
\hline Other & 6.7 & 6.0 & 7.7 & & \\
\hline None & 11.8 & 16.0 & 6.4 & & \\
\hline Did Not Respond & 2.2 & 3.0 & 1.3 & & \\
\hline Education Level & & & & 174 & $-4.17^{*}$ \\
\hline High School & 42.1 & 48.0 & 34.6 & & \\
\hline Some College & 33.7 & 43.0 & 21.8 & & \\
\hline B.A. or B.S. & 14.6 & 8.0 & 23.1 & & \\
\hline Some Graduate & 1.7 & 1.0 & 2.6 & & \\
\hline M.A. or M.S. & 5.6 & 0.0 & 12.8 & & \\
\hline Ph.D. & 1.1 & 0.0 & 2.6 & & \\
\hline Did not respond & 1.1 & 0.0 & 2.6 & & \\
\hline Difficulty Paying Bills & & & & 3 & $16.89^{*}$ \\
\hline None & 63.5 & 53.0 & 76.9 & & \\
\hline A Little & 19.1 & 28.0 & 7.7 & & \\
\hline Some & 10.7 & 14.0 & 6.4 & & \\
\hline A Great Deal & 2.8 & 4.0 & 1.3 & & \\
\hline Did Not Respond & 3.9 & 1.0 & 7.7 & & \\
\hline
\end{tabular}


Table 2

Group Differences on Study Variables for Younger and Older Adults

\begin{tabular}{lccccc}
\hline \multirow{2}{*}{ Variable } & Total Sample & Younger Adults & \multicolumn{1}{c}{ Older Adults } & & \\
\cline { 2 - 4 } & $M(S D)$ & $M(S D)$ & $M(S D)$ & $\underline{\mathrm{df}^{1}}$ & $\underline{\mathrm{t}}$ \\
\hline Age & $42.4(26.0)$ & $19.95(1.45)$ & $71.13(7.99)$ & 176 & $\mathrm{t}=-62.82^{* *}$ \\
Religiousness & $10.3(3.9)$ & $9.01(3.59)$ & $11.92(3.74)$ & 174 & $\mathrm{t}=-5.23^{*}$ \\
Spirituality & $34.1(5.3)$ & $34.05(5.41)$ & $34.16(5.14)$ & 173 & $\mathrm{t}=-.14$ \\
Self-Assessed Health & $9.1(1.2)$ & $9.09(1.21)$ & $9.13(1.14)$ & 176 & $\mathrm{t}=-.21$ \\
\hline${ }^{*} p<.01 .{ }^{* *} p<.001$. & & & & &
\end{tabular}

${ }^{1}$ Apparent differences in degrees of freedom are due to unequal variances. 
Table 3

Intercorrelations for the Sample Characteristics $(N=178)$

\begin{tabular}{lccccc}
\hline \multicolumn{1}{c}{ Measure } & Age & Gender $^{1}$ & Religiousness & Spirituality & $\begin{array}{c}\text { Self-Assessed } \\
\text { Health }\end{array}$ \\
\hline Age & -- & & & & \\
Gender & .10 & -- & & & \\
Religiousness & $.37^{* *}$ & .13 & -- & & \\
Spirituality & -.00 & .06 & $.30^{* *}$ & -- & - \\
Self-Assessed Health & .01 & -.12 & .02 & .09 & - \\
\hline${ }^{* *} p<.01$. & & & & &
\end{tabular}

${ }^{1}$ Correlations for gender are point-biserial. 
Table 4

Point-Biserial and Phi Coefficients of the sample Characteristics and the Five Decisional

Outcomes $(N=178)$

\begin{tabular}{lccccc}
\hline \multicolumn{1}{c}{$\begin{array}{c}\text { Decisional } \\
\text { Outcome }\end{array}$} & Age & Gender & Religiousness & Spirituality & $\begin{array}{c}\text { Self-Assessed } \\
\text { Health }\end{array}$ \\
\hline Fight Death & $-.21^{* *}$ & -.09 & -.15 & -.13 & -.00 \\
Accept Death & .08 & .08 & $.28^{* *}$ & $.26^{* *}$ & -.01 \\
Defer to Others & .09 & .05 & .12 & .02 & -.11 \\
Suicide & .06 & -.04 & $-.17^{*}$ & -.15 & .04 \\
Assisted Suicide &.- .10 & -.03 & $-.24^{* *}$ & $-.18^{*}$ & .04
\end{tabular}

${ }^{*} p<.05 .{ }^{* *} p<.01$. 
Table 5

Percent of Endorsement of End-of-Life Decisional Outcomes Between Younger ( $n=100$ and Older Adults ( $\mathrm{n}=78)$

\begin{tabular}{lccc}
\hline \multicolumn{1}{c}{ Decisional Outcomes } & $\begin{array}{c}\text { Total Sample } \\
\%(\mathrm{n})\end{array}$ & $\begin{array}{c}\text { Younger Adults } \\
\%(\mathrm{n})\end{array}$ & $\begin{array}{c}\text { Older Adults } \\
\%(\mathrm{n})\end{array}$ \\
\hline Fight Against Death ${ }^{*}$ & $5.6(10)$ & $10.3(10)$ & $0.0(0)$ \\
Accept Death & $70.2(125)$ & $73.2(71)$ & $78.3(54)$ \\
Defer the Decision to Others & $2.2(4)$ & $1.0(1)$ & $4.3(3)$ \\
End the Life through Suicide & $11.8(21)$ & $10.3(10)$ & $15.9(11)$ \\
End the Life through Assisted Suicide & $3.4(6)$ & $5.2(5)$ & $1.4(1)$ \\
${ }^{*} \chi^{2}(4, N=166)=7.57, p<.01$. & & &
\end{tabular}


Table 6

Percent of Endorsement of End-of-Life Decisional Outcomes Within the Older Adult

Group ( $n=78)$

\begin{tabular}{lcc}
\hline \multicolumn{1}{c}{ Decisional Outcome } & $\begin{array}{c}\text { Young Old (55 to 70) } \\
\%(\mathrm{n})\end{array}$ & $\begin{array}{c}\text { Old Old (71 to 84) } \\
\%(\mathrm{n})\end{array}$ \\
\hline Accept Death & $71.9(23)$ & $83.8(31)$ \\
Defer the Decision to Others & $6.3(2)$ & $2.7(1)$ \\
End the Life through Suicide & $18.8(6)$ & $13.5(5)$ \\
End the Life through Assisted Suicide & $3.1(1)$ & $0.0(0)$ \\
\hline Note. No older adults endorsed the decision of fight against death. Therefore it was not \\
included in this table.
\end{tabular}


Table 7

Summary of Multinomial Logistic Regression Analysis Predicting End-of-life Decisional

Outcomes

\begin{tabular}{|c|c|c|c|c|c|}
\hline Predictors and Outcomes & $\chi^{2}(4)$ & $\underline{\beta}$ & $\underline{\mathrm{SE}}$ & Odds Ratio & Wald Statistic \\
\hline Age & $9.34^{*}$ & & & & \\
\hline Fight against death & & -.10 & .07 & .90 & 1.84 \\
\hline Accept death & & -.01 & .02 & .99 & 19 \\
\hline Suicide & & .00 & .03 & 1.00 & .01 \\
\hline Assisted suicide & & -.02 & .04 & .98 & .43 \\
\hline Gender & .90 & & & & \\
\hline Fight against death & & -.93 & 1.39 & .39 & .45 \\
\hline Accept death & & -.31 & 1.22 & .73 & .07 \\
\hline Suicide & & -.34 & 1.30 & .72 & .07 \\
\hline Assisted suicide & & -.28 & 1.51 & .76 & .03 \\
\hline Religiousness & $11.33^{* *}$ & & & & \\
\hline Fight against death & & -.26 & .22 & .77 & 1.33 \\
\hline Accept death & & -.17 & .20 & .85 & .73 \\
\hline Suicide & & -.32 & .21 & .73 & 2.37 \\
\hline Assisted suicide & & $-.62^{\star *}$ & .28 & .54 & 4.70 \\
\hline Spirituality & 6.08 & & & & \\
\hline Fight against death & & -.12 & .15 & .90 & .55 \\
\hline Accept death & & .00 & .13 & 1.00 & .00 \\
\hline
\end{tabular}




\section{(Table 7 continued)}

\begin{tabular}{lccccc}
\hline Predictors and Outcomes & $\chi^{2}(4)$ & $\underline{\beta}$ & $\underline{\text { SE }}$ & Odds Ratio & Wald Statistic \\
\hline Suicide & & -.08 & .14 & .93 & .31 \\
Assisted suicide & & -.12 & .15 & .89 & .65 \\
Self-Assessed Health & 2.83 & & & & \\
Fight against death & .63 & .50 & 1.87 & 1.57 \\
Accept death & .52 & .41 & 1.68 & 1.61 \\
Suicide & .68 & .46 & 1.97 & 2.19 \\
Assisted Suicide & .84 & .56 & 2.32 & 2.30 \\
\end{tabular}

${ }^{\star} p=.05 .{ }^{* \star} p<.05$. 
Table 8

2(Age) $\times 3$ (Domain) $\times 7$ (Strategy) Repeated Measures Analysis of Covariance-

Between- and Within-Subjects Results

\begin{tabular}{|c|c|c|c|c|c|}
\hline Source & $d f$ & SS & MS & $F$ & $p$ \\
\hline \multicolumn{6}{|l|}{ Between-Subjects Effects } \\
\hline Age $(A)$ & 1 & .03 & .03 & .15 & .70 \\
\hline Religiousness (R) & 1 & .00 & .00 & .02 & .89 \\
\hline Spirituality (Sp) & 1 & 1.21 & 1.21 & $5.27^{*}$ & .02 \\
\hline Error & 151 & 34.58 & .23 & & \\
\hline \multicolumn{6}{|l|}{ Within-Subjects Effects } \\
\hline Domain (D) & 2 & .63 & .31 & $3.16^{*}$ & .04 \\
\hline$D \times A$ & 2 & .08 & .04 & .44 & .65 \\
\hline$D \times R$ & 2 & .00 & .00 & .03 & .97 \\
\hline$D \times S p$ & 2 & .78 & .39 & $3.94^{*}$ & .02 \\
\hline Error (SC) & 302 & 29.95 & .09 & & \\
\hline Strategy (ST) & 6 & 10.73 & 1.79 & $6.71^{* * *}$ & .00 \\
\hline ST $\times A$ & 6 & 17.18 & 2.86 & $10.74^{* * *}$ & .00 \\
\hline $\mathrm{ST} \times \mathrm{R}$ & 6 & 1.75 & .29 & 1.10 & .36 \\
\hline ST $x$ Sp & 6 & 2.24 & .37 & 1.40 & .21 \\
\hline Error (ST) & 906 & 241.45 & .27 & & \\
\hline Domain $(D) \times$ Strategy $(\mathrm{ST})$ & 12 & 6.78 & .57 & $2.42^{\star *}$ & .00 \\
\hline$D \times S T \times A$ & 12 & 4.12 & .34 & 1.47 & .13 \\
\hline
\end{tabular}


(Table 8 continued)

\begin{tabular}{llccccc}
\hline Source & & $d f$ & SS & MS & $F$ & $p$ \\
\hline & & 12 & 6.48 & .54 & $2.31^{* *}$ & .00 \\
& D x ST x R & 12 & 3.27 & .27 & 1.17 & .30 \\
Error (D x ST) & 1812 & 423.21 & .23 & &
\end{tabular}

Note. $A=$ age; $R=$ religiousness; $S p=$ spirituality

${ }^{*} p<.05 .{ }^{* *} p<.01 .{ }^{* * *} p<.001$. 
Table 9

Domain x Strategy Interaction-Means, Standard Deviations, and Post hoc tests

\begin{tabular}{lcccccc}
\hline \multirow{1}{c}{ Strategies } & & Domain 1 & Domain 2 & Domain 3 & \\
& Across Domains & End-of-Life & Family & Consumer & $\mathrm{F}$ \\
& $M(\mathrm{SD})$ & $M(\mathrm{SD})$ & $M(\mathrm{SD})$ & $M(\mathrm{SD})$ & $\mathrm{df}(2,152)$ \\
\hline Religion $^{1}$ & $.3910(.69)$ & $.3713(.67)$ & $0(0)$ & $0(0)$ & $5.98^{\star *}$ \\
Change Self Cognition & & $.3910(.66)$ & $.2096(.42)$ & $.0479(.29)$ & $.1313(.34)$ & $5.46^{* *}$ \\
Change Self Behavior & $1.846(1.29)$ & $.7186(.77)$ & $.7066(.71)$ & $.4188(.62)$ & 2.41 \\
Change Other Cognition & $.4231(.58)$ & $.024(.19)$ & $.3234(.48)$ & $.0563(.23)$ & 1.53 \\
Change Other Behavior & $.3654(.57)$ & $.012(.11)$ & $.3413(.51)$ & $.0188(.14)$ & 0.31 \\
Seek Social Support & $.4936(.78)$ & $.4192(.68)$ & $.0719(.34)$ & $.0188(.18)$ & 1.87 \\
Seek Formal Support & $1.750(1.44)$ & $.3234(.62)$ & $.2455(.52)$ & $1.18(.90)$ & 0.44 \\
\hline
\end{tabular}

${ }^{* *} p<.01$.

${ }^{1} D_{1}>D_{2}, D_{3}, t(163)=7.16, p<.001$. No other t-test results because of zero values in domains 2 and 3.

${ }^{2} \mathrm{D}_{1}>\mathrm{D}_{3}>\mathrm{D}_{2} .(\mathrm{D} 1-\mathrm{D} 2, \mathrm{t}(163)=4.23, \mathrm{p}<.001) ;(\mathrm{D} 1-\mathrm{D} 3, \mathrm{t}(157)=2.10, \mathrm{p}<.05)$; (D3-D2, $t(157)=2.23, p<.05)$. 
Table 10

Strategy x Age Interaction-Means of Strategies Generated by the Participants Across Three Decisional Domains

\begin{tabular}{lcccccc}
\hline \multirow{2}{*}{ Strategy Type } & Total Sample & Younger Adults & Older Adults & & \\
\cline { 2 - 3 } & $M(\mathrm{SD})$ & $M(\mathrm{SD})$ & & $M(\mathrm{SD})$ & $\underline{\mathrm{df}^{1}}$ & $\underline{\mathrm{t}}$ \\
\hline Religion & $.39(.69)$ & $.32(.68)$ & $.48(.68)$ & 154 & -1.47 \\
Change Self Cognition & $.39(.66)$ & $.54(.75)$ & $.18(.43)$ & 145 & $3.82^{* * *}$ \\
Change Self Behavior & $1.85(1.29)$ & $1.97(1.09)$ & $1.68(1.51)$ & 111 & $1.31^{* *}$ \\
Change Other Cognition & $.42(.58)$ & $.49(.59)$ & $.33(.56)$ & 154 & 1.67 \\
Change Other Behavior & $.37(.57)$ & $.44(.64)$ & $.26(.44)$ & 153 & $2.16^{*}$ \\
Seek Social Support & $.49(.78)$ & $.54(.84)$ & $.42(.70)$ & 154 & .95 \\
Seek Formal Support & $1.75(1.44)$ & $1.28(1.07)$ & $2.39(1.62)$ & 105 & $-4.88^{* * *}$ \\
\hline
\end{tabular}

${ }^{*} p<.05 .{ }^{* *} p<.01 .{ }^{* \star *} p<.001$.

${ }^{1}$ Apparent differences in degrees of freedom due to unequal variances. 
Table 11

Domain x Spirituality Interaction—Strategies Generated within Problem Domains for Each Level of Spirituality

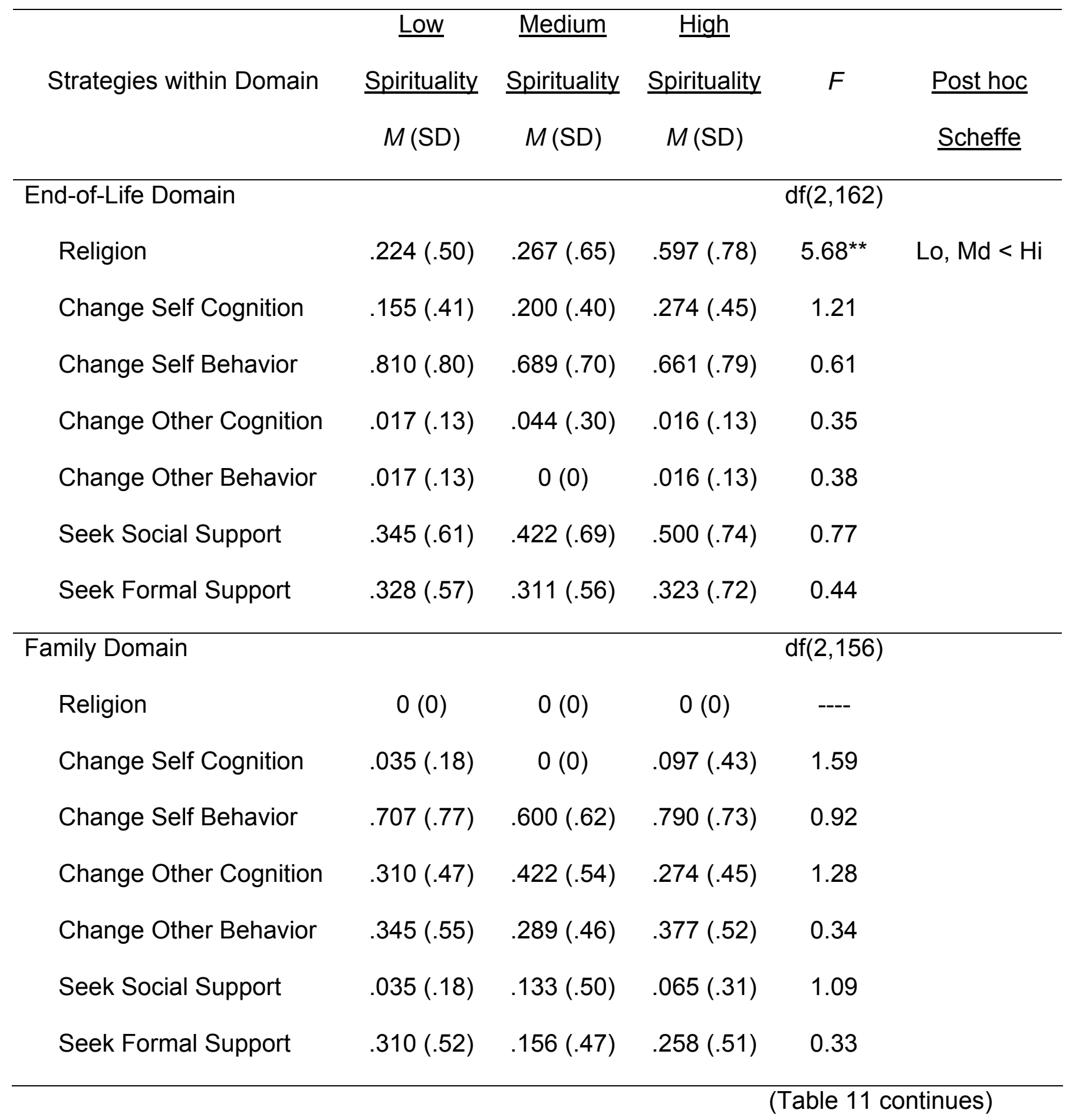


(Table 11 continued)

\begin{tabular}{|c|c|c|c|c|c|}
\hline \multirow{3}{*}{ Strategies within Domain } & Low & Medium & $\underline{\text { High }}$ & \multirow{3}{*}{$F$} & \\
\hline & Spirituality & Spirituality & Spirituality & & Post hoc \\
\hline & $M(\mathrm{SD})$ & $M(\mathrm{SD})$ & $M(\mathrm{SD})$ & & Scheffe \\
\hline Consumer Domain & & & & $\mathrm{df}(2,156)$ & \\
\hline Religion & $0(0)$ & $0(0)$ & $0(0)$ & ---- & \\
\hline Change Self Cognition & $.071(.26)$ & $.070(.26)$ & $.233(.43)$ & $4.47^{*}$ & $\mathrm{Lo}<\mathrm{Hi}$ \\
\hline Change Self Behavior & $.536(.69)$ & $.326(.52)$ & $.367(.61)$ & 1.71 & \\
\hline Change Other Cognition & $.030(.19)$ & $.070(.26)$ & $.067(.25)$ & 0.35 & \\
\hline Change Other Behavior & $0(0)$ & $.047(.21)$ & $.015(.13)$ & 1.43 & \\
\hline Seek Social Support & $0(0)$ & $0(0)$ & $.050(.29)$ & 1.50 & \\
\hline Seek Formal Support & $1.19(.94)$ & $1.14(.86)$ & $1.22(.88)$ & 0.09 & \\
\hline
\end{tabular}

${ }^{*} p<.05 .{ }^{* *} p<.01$. 
Table 12

Most Common Strategies Generated by Participants Within Each Decisional Domain

Decisional Domain

$\%(n)$

End-of-Life Domain

Religion

Change Self Behavior

Seek Social Support

Seek Formal Support

Family Domain

Change Self Behavior

Change Other Cognition

Change Other Behavior

Seek Formal Support

Consumer Domain

Change Self Cognition

Change Self Behavior

Seek Formal Support

Change Other Cognition
$25.8(46)$

$53.4(95)$

$29.8(53)$

$23.6(42)$

$54.0(96)$

$29.8(53)$

$30.4(54)$

$19.1(34)$

11.8 (21)

$32.1(57)$

70.8 (126)

$5.1(9)$
$21.0(21)$

$64.0(64)$

$35.0(35)$

$15.0(15)$

$32.1(25)$

$39.8(31)$

$23.0(18)$

$34.7(27)$

$\%(n)$

$\%(n)$

$\%(n)$ Younger Adults Older Adults 


\title{
Appendix A: Informed Consent Form-Younger Adults
}

\section{Consent and Information Form}

\section{Religiousness and End-of-Life Decisions}

\author{
Introduction
}

I, have been invited to participate in this research study which has been explained to me by Loriann Sonntag or her research assistant. This research is being conducted by Loriann Sonntag, Siwar Bizri, and Julie Patrick, Ph.D. to fulfill the requirements for a master's thesis in developmental psychology in the Department of Psychology at West Virginia University, under the supervision of Julie Patrick, Ph.D.

Purposes of the Study The purpose of this study is to examine the associations among religiousness and spirituality and hypothetical end-of-life decisions.

Description of Procedures

This study involves a pencil and paper survey format and will take approximately one hour for me to complete. I have been told to fill out nine questionnaires about religiousness and spirituality and hypothetical decisions. I have also been told that I may see the questionnaire before signing this consent form and that I do not have to answer all of the questions if I decide to participate. Approximately 100 subjects are expected to participate in this study.

For more information about this research, I can contact Loriann Sonntag, at 304-2932001, or her supervisor, Dr. Julie Patrick, at 304-293-2001, x31680. For information regarding my rights as a research subject, I may contact the Office of Research Compliance at 304-293-7073.

Risks and Discomforts I understand that there is no physical health risk associated with my participation in this study. However, I also understand that my participation may include a minimal risk of emotional distress as I encounter the hypothetical end-of-life situation in the decision vignette.

May 2004

Page 1 of 2 


\section{Consent and Information Form}

\section{Religiousness and End-of-Life Decisions}

Intervention

Should I encounter emotional distress during my participation in this study, I understand that intervention will be in the form of a referral to the Carruth Center.

Confidentiality

I understand that any information about me obtained as a result of my participation in this research will be kept as confidential as legally possible. I understand that my research records and test results, just like hospital records, may be subpoenaed by court order or may be inspected by the study sponsor or federal regulatory authorities (including the FDA if applicable) without my additional consent. In any publications that result from this research, neither my name nor any information from which I might be identified will be published without my consent.

Voluntary Participation Participation in this study is voluntary. I understand that I am free to withdraw my consent to participate in this study at any time. Refusal to participate or withdrawal from this study will involve no penalty to me. I have been given the opportunity to ask questions about the research, and I have received answers concerning areas I did not understand. In the event that new information becomes available that may affect my willingness to continue to participate in the study, this information will be given to me so I may make an informed decision about my participation.

Upon signing the form, I will receive a copy.

I willingly consent to participate in this research.

$\overline{\text { Signature of Subject or Subject's Legal Representative }}$

Signature of Investigator or Investigator's Representative

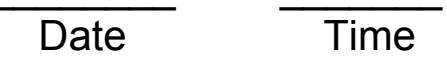

Date

Time

May 2004

Page 2 of 2

Initials $\quad$ Date 
Dear Sir or Madam:

My name is Loriann Sonntag and I am a second-year graduate student in the Life-Span Developmental Psychology program at West Virginia University. I am currently conducting research for my Master's Thesis entitled "Religiousness and Spirituality in Decision Making Processes and Outcomes", which is being conducted under the guidance of my advisor, Dr. Julie Patrick. The reason for this letter is to ask for your help in completing this project. Enclosed with this letter you will find an anonymous survey packet and two informed consent forms. Please consider filling out the survey, signing one of the consent forms and mailing them back in the postage paid envelope that has been provided for you.

Your participation in this research would only involve completing the enclosed survey. No further contact would be made concerning this particular research study. Please remember that your participation is completely voluntary and you are allowed to skip any questions on the survey that you do not feel comfortable answering. If you would like to participate, please complete the survey, sign one of the informed consent forms and return both to me in the provided envelope. If you choose not to participate, I would appreciate it if you would consider passing the booklet and forms along to a friend, neighbor, or relative.

If you have any questions or concerns, please call Dr. Patrick at (304) 293-2001, x 31680 or toll-free at 1-866-535-3343, x 31680.

Sincerely,

Loriann Sonntag, B.A.

Julie Hicks Patrick, Ph.D.

Assistant Professor, Life-Span Development

May 2004 
Appendix C: Informed Consent Form—Older Adults

Consent and Information Form

Religiousness and End-of-Life Decisions

Introduction

I, have been invited to participate in this research study which has been explained to me by Loriann Sonntag or her research assistant. This research is being conducted by Loriann Sonntag, Siwar Bizri, and Julie Patrick, Ph.D. to fulfill the requirements for a master's thesis in developmental psychology in the Department of Psychology at West Virginia University, under the supervision of Julie Patrick, Ph.D.

Purposes of the Study

The purpose of this study is to examine the associations among religiousness and spirituality and hypothetical end-of-life decisions.

Description of Procedures

This study involves a pencil and paper survey format and will take approximately one hour for me to complete. I have been told to fill out nine questionnaires about religiousness and spirituality and hypothetical decisions. I have also been told that I may see the questionnaire before signing this consent form and that I do not have to answer all of the questions if I decide to participate. Approximately 100 subjects are expected to participate in this study.

For more information about this research, I can contact Loriann Sonntag, at 304-2932001, or her supervisor, Dr. Julie Patrick, at 304-293-2001, x31680. For information regarding my rights as a research subject, I may contact the Office of Research Compliance at 304-293-7073.

Risks and Discomforts I understand that there is no physical health risk associated with my participation in this study. However, I also understand that my participation may include a minimal risk of emotional distress as I encounter the hypothetical end-of-life situation in the decision vignette.

May 2004

Page 1 of 2

Initials

Date 


\section{Consent and Information Form}

\section{Religiousness and End-of-Life Decisions}

Intervention

Should I encounter emotional distress during my participation in this study, I understand that intervention will be in the form of a referral to the Quin Curtis Center Center.

Confidentiality

I understand that any information about me obtained as a result of my participation in this research will be kept as confidential as legally possible. I understand that my research records and test results, just like hospital records, may be subpoenaed by court order or may be inspected by the study sponsor or federal regulatory authorities (including the FDA if applicable) without my additional consent. In any publications that result from this research, neither my name nor any information from which I might be identified will be published without my consent.

Voluntary Participation Participation in this study is voluntary. I understand that I am free to withdraw my consent to participate in this study at any time. Refusal to participate or withdrawal from this study will involve no penalty to me. I have been given the opportunity to ask questions about the research, and I have received answers concerning areas I did not understand. In the event that new information becomes available that may affect my willingness to continue to participate in the study, this information will be given to me so I may make an informed decision about my participation.

Upon signing the form, I will receive a copy.

I willingly consent to participate in this research.

$\overline{\text { Signature of Subject or Subject's Legal Representative }}$

Signature of Investigator or Investigator's Representative

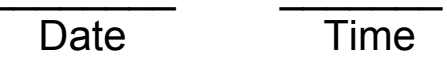

Date

Time

May 2004

Page 2 of 2

Initials Date 


\section{Appendix D: Decision Vignettes}

\section{Decision Vignettes}

\section{High-Emotional Salience}

1. A 75-year-old man has just been told by his doctor that he has less than one year to live. The last several months of his illness will likely be very painful. The man is thinking about committing suicide. What should the man do?

\section{Medium-Emotional Salience}

2. A person has an 86-year-old grandmother who drives a car and likes to feel mobile and independent. But the person feels that the grandmother is physically incapable of driving safely, and that her driving is a danger both to herself and to the public. What should the person do?

\section{Low-Emotional Salience}

3. A person bought some things for her daughters that turned out to be clearly defective. She tries to return them to the store, but the manager will do nothing about it. What should the woman do? 
Appendix E: Pilot Study

Vignette Ratings of Emotional Salience

Please take the time to answer the following questions.

Gender: Male Female

Age: $\_25$

Please read each of the following vignettes and rate them according to the scales that follow.

1. A 25-year-old man has just been told by his doctor that he has less than one year to live. The last several months of his illness will likely be very painful. The man is thinking about committing suicide. What should the man do?

a. To what degree would you describe this decision as:

\begin{tabular}{cccccc}
\hline & Not at all & A little & Some & Very & Extremely \\
\hline Emotional? & 1 & 2 & 3 & 4 & 5 \\
Common/Typical? & 1 & 2 & 3 & 4 & 5 \\
Difficult? & 1 & 2 & 3 & 4 & 5 \\
Important? & 1 & 2 & 3 & 4 & 5 \\
\hline
\end{tabular}

b. To what extent did the story provide enough information to allow you to make a good decision?

1

Not enough information
2

Some information
3 Sufficient Information 
2. A person has an 86-year-old grandmother who drives a car and likes to feel mobile and independent. But the person feels that the grandmother is physically incapable of driving safely, and that her driving is a danger both to herself and to the public. What should the person do?**

a. To what degree would you describe this decision as:

\begin{tabular}{cccccc}
\hline & Not at all & A little & Some & Very & Extremely \\
\hline Emotional? & 1 & 2 & 3 & 4 & 5 \\
Common/Typical? & 1 & 2 & 3 & 4 & 5 \\
Difficult? & 1 & 2 & 3 & 4 & 5 \\
Important? & 1 & 2 & 3 & 4 & 5 \\
\hline
\end{tabular}

b. To what extent did the story provide enough information to allow you to make a good decision?

1

Not enough information Some information Sufficient Information

3. A person bought some things for her daughters that turned out to be clearly defective. She tries to return them to the store, but the manager will do nothing about it. What should the woman do?**

a. To what degree would you describe this decision as:

\begin{tabular}{cccccc}
\hline & Not at all & A little & Some & Very & Extremely \\
\hline Emotional? & 1 & 2 & 3 & 4 & 5 \\
Common/Typical? & 1 & 2 & 3 & 4 & 5 \\
Difficult? & 1 & 2 & 3 & 4 & 5 \\
Important? & 1 & 2 & 3 & 4 & 5 \\
\hline
\end{tabular}

b. To what extent did the story provide enough information to allow you to make a good decision?

1

Not enough information
2

Some information
3

Sufficient Information 
4. A 65-year-old woman lives alone in her third floor apartment in the city. She has few visitors and rarely goes out. She complains that she misses being with others and that she doesn't want to be a burden to her family. What should the woman do?

a. To what degree would you describe this decision as:

\begin{tabular}{cccccc}
\hline & Not at all & A little & Some & Very & Extremely \\
\hline Emotional? & 1 & 2 & 3 & 4 & 5 \\
Common/Typical? & 1 & 2 & 3 & 4 & 5 \\
Difficult? & 1 & 2 & 3 & 4 & 5 \\
Important? & 1 & 2 & 3 & 4 & 5 \\
\hline
\end{tabular}

b. To what extent did the story provide enough information to allow you to make a good decision?

1

Not enough information
2

Some information
3

Sufficient Information

5. A 70-year-old widow has six children who live out of state, while she lives in the suburb of a large city where she has lived for the past 52 years. She likes the young families moving into the neighborhood, but wants to interact more with people her own age. What should the woman do?

1. To what degree would you describe this decision as:

\begin{tabular}{cccccc}
\hline & Not at all & A little & Some & Very & Extremely \\
\hline Emotional? & 1 & 2 & 3 & 4 & 5 \\
Common/Typical? & 1 & 2 & 3 & 4 & 5 \\
Difficult? & 1 & 2 & 3 & 4 & 5 \\
Important? & 1 & 2 & 3 & 4 & 5 \\
\hline
\end{tabular}

2. To what extent did the story provide enough information to allow you to make a good decision?

1

Not enough information
2

Some information
3

Sufficient Information 
6. A 75-year-old man has just been told by his doctor that he has less than one year to live. The last several months of his illness will likely be very painful. The man is thinking about committing suicide. What should the man do?**

a. To what degree would you describe this decision as:

\begin{tabular}{cccccc}
\hline & Not at all & A little & Some & Very & Extremely \\
\hline Emotional? & 1 & 2 & 3 & 4 & 5 \\
Common/Typical? & 1 & 2 & 3 & 4 & 5 \\
Difficult? & 1 & 2 & 3 & 4 & 5 \\
Important? & 1 & 2 & 3 & 4 & 5 \\
\hline
\end{tabular}

b. To what extent did the story provide enough information to allow you to make a good decision?

1

Not enough information

Some information

3 Sufficient Information

Note: ${ }^{* *}$ vignettes used in the current study. 


\section{Appendix F: Demographics Questionnaire}

\section{Demographics Questionnaire}

1. What is your age in years?

2. What is your marital status, are you:

$\square$ Married

Widowed

Divorced $\square$ Single, Never married, or

$\square$ Other (please specify below)

3. What is your religion, are you:

$\begin{array}{ll}\square \text { Protestant } \square \text { Catholic } & \square \text { Jewish, or } \\ \square \text { Some other religion (please specify) } & \square \text { None }\end{array}$

4. Do you currently live:
Alone,
With a spouse, or
With Others? (please specify)

5. To which racial group do you belong?

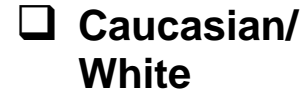

White
$\square$ African Americanl Black

$\square$ Other (Please specify)
6. Are you:
Male or
Female?

7. What is the highest level of education you've completed?

8. How much difficulty do you have paying your bills? Would you say:
A great deal of difficulty
Some difficulty
No difficulty, or 
9. Please circle the letter that describes the total income last year that you (\& household members) received from all sources. Be sure to include Social Security, pensions, bank interest, reparations, annuities, and so forth:

$\begin{array}{lll}\square \text { A < 10K } & \square \text { E 25-30 K } & \square \text { I 45-50 K } \\ \square \text { B 10-15 K } & \square \text { F 30-35 K } & \square \text { J 50-55 K } \\ \square \text { C 15-20 K } & \square \text { G 35-40 K } & \square \text { K 55-60 K } \\ \square \text { D 20-25 K } & \square \text { H 40-45 K } & \square \text { L 60-65 K }\end{array}$

\begin{tabular}{l}
$\square$ M 65-70 K \\
\hline N 70-75 K \\
\hline O 75+ K \\
$\square$ P: PREFER NOT \\
TO ANSWER
\end{tabular}


Appendix G: Self-Assessed Health Scale

\section{Self-Assessed Health}

1. How would you rate your overall health at the present time? Is it:

$\square$ Excellent $\square$ Good $\square$ Fair, or $\square$ Poor?

2. Is your health now better, about the same, or not as good as it was 3 years ago?

$\square$ Better $\square$ The Same, or $\square$ Not as Good?

3. Do your health problems stand in the way of your doing the things you want to do?

$\square$ Not at all $\square$ A little, or $\square$ A great deal?

4. Compared with most other people your age, would you say your health is:

$\square$ Better $\square$ The Same, or $\square$ Not as Good? 


\section{Appendix H: Organizational Religiousness}

\section{Religious Activities}

1. I attend religious services. ......

2. I attend religious meetings or missions. . . . . . . . . . . . . .

VERY SOMETIMES OFTEN

VERY OFTEN

3. I contribute money to my church or synagogue. ............

4. I take part in various religious organizations............

\section{VERY SOMETIMES}

OFTEN

VERY

OFTEN
ALMOST NEVER NEVER

ALMOST NEVER NEVER

ALMOST NEVER NEVER

ALMOST NEVER NEVER 
Appendix I: Spiritual Transcendence Scale

Spiritual Transcendence Scale—Universality Subscale

1. I feel that on a higher level all of us share a common bond.

Strongly

Agree

Agree

Neutral

Strongly

Disagree

2. All life is interconnected.

Strongly

Agree

Agree

Neutral

Disagree

Strongly

Disagree

3. There is a higher plane of consciousness or spirituality that binds all people.

Strongly

Agree

Agree

Neutral

Disagree

Strongly Disagree

4. Although individual people may be difficult, I feel an emotional bond with all of humanity.

Strongly

Agree

Agree

Neutral

Disagree

Strongly

Disagree

5. I do not believe that there is a larger meaning to life.

Strongly

Agree

Neutral

Disagree

Strongly

Agree

6. I do not believe that death is a doorway to another plane of existence.

Strongly

Agree

Agree

Neutral

Disagree

Strongly

Disagree

7. I do not believe there is a larger plan to life.

Strongly

Agree

Agree

Neutral

Disagree

Strongly

Disagree 
8. There is an order to the universe that transcends human thinking.

Strongly

Agree

Agree

Neutral

Disagree

Strongly

Disagree

9. I believe that on some level my life is intimately tied to all of human kind.

Strongly

Agree

Agree

Neutral

Disagree

Strongly

Disagree 
Appendix J: Coding Sheet

RID \# Strategies/Outcomes Coding Sheet

\begin{tabular}{|c|c|c|c|c|c|c|}
\hline & D-1a & D-2a & D-3a & D-4a & D-5a & D-6a \\
\hline \multicolumn{7}{|l|}{ Strategies } \\
\hline \multicolumn{7}{|l|}{ Religion } \\
\hline \multicolumn{7}{|l|}{ Cooperate/Discuss } \\
\hline \multicolumn{7}{|l|}{ Seek Information } \\
\hline \multicolumn{7}{|l|}{ Change Self (emotion) } \\
\hline \multicolumn{7}{|l|}{ Change Self (Cognition) } \\
\hline \multicolumn{7}{|l|}{ Change Self (BEHAVIOR) } \\
\hline \multicolumn{7}{|l|}{ Change Other (emotion) } \\
\hline \multicolumn{7}{|l|}{ Change Other (Cognition) } \\
\hline \multicolumn{7}{|l|}{ Change Other (BEHAVIOR) } \\
\hline \multicolumn{7}{|l|}{ Change the Environment } \\
\hline \multicolumn{7}{|l|}{ Change to a new Environment } \\
\hline \multicolumn{7}{|l|}{ Seek Social Support } \\
\hline \multicolumn{7}{|l|}{ Seek Formal Support } \\
\hline \multicolumn{7}{|l|}{ Outcomes } \\
\hline \multicolumn{7}{|l|}{ Fight against Death } \\
\hline \multicolumn{7}{|l|}{ Accept Death } \\
\hline \multicolumn{7}{|l|}{ Defer the decision to others } \\
\hline \multicolumn{7}{|l|}{ End the life through suicide } \\
\hline End the life through assisted suicide & & & & & & \\
\hline
\end{tabular}


Appendix K: Problem-Solving Strategies/Decisional Outcomes Coding Manual (Adapted from Swenson, 2003)

\section{Problem-Solving Strategies}

Religion: Any thoughts, actions, or behaviors suggested by the respondent that involve turning toward religion or religious beliefs (e.g., pray; discuss the situation with your minister; go to church).

Cooperate/Discuss: All strategies suggesting that others be brought into a discussion about the decision with the exception of discussions with religious clergy (e.g., talk with your family; discuss other options with a doctor).

Seek Information: All strategies suggesting that the individual take the time to seek additional information about their current situation (e.g., seek a second opinion; consult the internet for further information).

Change Self Emotion: Strategies suggesting a change in the individual's emotion in response to the situation (e.g., think positively; try to enjoy your remaining moments).

Change Self Cognition: Strategies suggesting a change in the individual's thought process in response to the situation (e.g., realizing the he or she is not a burden to their family or society; forget about the dresses and move on).

Change Self Behavior: Strategies suggesting specific actions or behaviors for the individual solving the problem, with the exception of religious behaviors (e.g., shop at a different store; go visit your family; volunteer in the community; drive for the grandmother; discuss the situation with your family). 
Change Other Emotion: Strategies suggesting a change in emotion for other individuals involved in the situation (e.g., help the grandmother to feel needed; tell your family not to worry).

Change Other Cognition: Strategies suggesting a change in thought for other individuals involved in the decision process (e.g., convince the grandmother she needs to stop driving; assist family members in understanding that the decision is not theirs to make).

Change Other Behavior: Strategies suggesting specific actions or behaviors for other individuals involved in the problem situation, with the exception of religious behaviors (e.g., tell everyone to shop at another store; Grandma should stop driving; have your family come and visit).

Change the Environment: Strategies suggesting a physical change to the individual's existing environment (e.g., build a ramp onto the house; bring in a hospital bed).

Change to a New Environment: Strategies suggesting that, in order to solve the problem, the individual should move to a different environment (e.g., move to Florida; move in with your family; go to a nursing home).

Seek Social Support: Strategies suggesting that the individual should rely on family and/or friends for assistance (e.g., talk to your family; ask your friends for advice).

Seek Formal Support: Strategies suggesting that the individual turn to professionals for support, with the exception of religious clergy (e.g., get a second opinion from another doctor; call a nurse for help; call in Hospice; ask to speak to a manager). 


\section{Decisional Outcomes}

Fight Against Death: Final decisions specifying that the individual do all they can to fight the diagnosis of death (e.g., get a second opinion; explore all available treatments; don't give up).

Accept Death: Final decisions specifying that the individual resign themselves to the diagnosis and prepare for death (e.g., make things right with God; get your affairs in order; say goodbye to your family).

Defer the Decision to Others: Final decisions specifying that the individual rely on other individuals to make the decision for them (e.g., ask your family to make the decision for you; ask you doctor to make the decision regarding treatment).

End the Life through Suicide: Final decisions specifying that the individual should use any means to end their own life (e.g., understand that there is no other alternative; give up and find a way to leave this world; say your goodbyes and then take those pills).

End the Life through Assisted Suicide: Final decisions specifying that the individual should seek someone's help in ending their life (e.g., ask a doctor for a lethal injection; find someone close to you to help you end your life). 